\title{
The hot core towards the intermediate-mass protostar NGC 7129 FIRS 2
}

\section{Chemical similarities with Orion $\mathrm{KL}^{\star, \star \star, \star \star \star}$}

\author{
A. Fuente ${ }^{1}$, J. Cernicharo ${ }^{2}$, P. Caselli ${ }^{3}$, C. $\mathrm{M}^{\mathrm{c}} \mathrm{Coey}^{4}$, D. Johnstone ${ }^{5,6,7}$, M. Fich ${ }^{4}$, T. van Kempen ${ }^{8}$, Aina Palau ${ }^{9}$, \\ U. A. Yildiz ${ }^{10}$, B. Tercero ${ }^{2}$, and A. López ${ }^{2}$ \\ ${ }^{1}$ Observatorio Astronómico Nacional (OAN, IGN), Apdo 112, 28803 Alcalá de Henares, Spain \\ e-mail: a.fuente@oan.es \\ 2 Instituto de Ciencia de Materiales de Madrid (ICMM) C/ Sor Juana Inés de la Cruz 3, Cantoblanco, 28049 Madrid, Spain \\ 3 Max Planck Institute for Extraterrestrial Physics, Postfach 1312, 85741 Garching, Germany \\ ${ }^{4}$ Department of Physics and Astronomy, University of Waterloo, Waterloo, Ontario, N2L 3G1, Canada \\ 5 Department of Physics \& Astronomy, University of Victoria, Victoria, BC, V8P 1A1, Canada \\ ${ }^{6}$ National Research Council of Canada, Herzberg Institute of Astrophysics, 5071 West Saanich Road, Victoria, BC, V9E 2E7, \\ Canada \\ 7 Joint Astronomy Centre, 660 North A'ohoku Place, University Park, Hilo, HI 96720, USA \\ ${ }^{8}$ Leiden Observatory, Leiden University, PO Box 9513, 2300 RA Leiden, The Netherlands \\ ${ }^{9}$ Centro de Radioastronomía y Astrofísica, Universidad Nacional Autónoma de Mexico, PO Box 3-72, 58090 Morelia, Michoacán, \\ Mexico \\ 10 Jet Propulsion Laboratory, California Institute of Technology, 4800 Oak Grove Drive, Pasadena, CA 91109, USA
}

Received 18 November 2013 / Accepted 17 May 2014

\section{ABSTRACT}

\begin{abstract}
Context. This paper is dedicated to the study of the chemistry of the intermediate-mass (IM) hot core NGC 7129 FIRS 2 , probably the most compact warm core found in the $2-8 M_{\odot}$ stellar mass range.

Aims. Our aim is to determine the chemical composition of the IM hot core NGC 7129 FIRS 2, and to provide new insights on the chemistry of hot cores in a more general context.

Methods. NGC 7129 FIRS 2 (hereafter, FIRS 2) is located at a distance of $1250 \mathrm{pc}$ and high spatial resolution observations are required to resolve the hot core at its center. We present a molecular survey from $218200 \mathrm{MHz}$ to $221800 \mathrm{MHz}$ carried out with the IRAM Plateau de Bure Interferometer (PdBI). These observations were complemented with a long integration single-dish spectrum taken with the IRAM $30 \mathrm{~m}$ telescope in Pico de Veleta (Spain). We used a local thermodynamic equilibrium (LTE) single temperature code to model the whole dataset.

Results. The interferometric spectrum is crowded with a total of $\approx 300$ lines from which a few dozen remain unidentified. The spectrum has been modeled with a total of 20 species and their isomers, isotopologues, and deuterated compounds. Complex molecules like methyl formate $\left(\mathrm{CH}_{3} \mathrm{OCHO}\right)$, ethanol $\left(\mathrm{CH}_{3} \mathrm{CH}_{2} \mathrm{OH}\right)$, glycolaldehyde $\left(\mathrm{CH}_{2} \mathrm{OHCHO}\right)$, acetone $\left(\mathrm{CH}_{3} \mathrm{COCH}_{3}\right)$, dimethyl ether $\left(\mathrm{CH}_{3} \mathrm{OCH}_{3}\right)$, ethyl cyanide $\left(\mathrm{CH}_{3} \mathrm{CH}_{2} \mathrm{CN}\right)$, and the aGg' conformer of ethylene glycol $\left(\mathrm{aGg}\right.$ ' $\left.\left(\mathrm{CH}_{2} \mathrm{OH}\right)_{2}\right)$ are among the detected

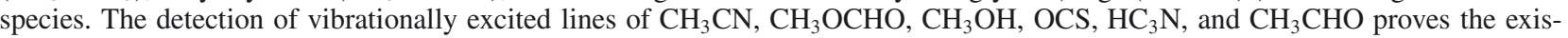
tence of gas and dust at high temperatures. The gas kinetic temperature estimated from the vibrational lines of $\mathrm{CH}_{3} \mathrm{CN}_{2} \sim 405_{-67}^{+100} \mathrm{~K}$, is similar to that measured in massive hot cores. Our data allow an extensive comparison of the chemistry in FIRS 2 and the Orion hot core.

Conclusions. We find a quite similar chemistry in FIRS 2 and Orion. Most of the studied fractional molecular abundances agree within a factor of 5. Larger differences are only found for the deuterated compounds $\mathrm{D}_{2} \mathrm{CO}$ and $\mathrm{CH}_{2} \mathrm{DOH}$ and a few molecules $\left(\mathrm{CH}_{3} \mathrm{CH}_{2} \mathrm{CN}, \mathrm{SO}_{2}, \mathrm{HNCO}\right.$ and $\left.\mathrm{CH}_{3} \mathrm{CHO}\right)$. Since the physical conditions are similar in both hot cores, only different initial conditions (warmer pre-collapse and collapse phase in the case of Orion) and/or different crossing times of the gas in the hot core can explain this behavior. We discuss these two scenarios.
\end{abstract}

Key words. astrochemistry - stars: formation - ISM: individual objects: NGC 7129 FIRS 2

\section{Introduction}

* Based on observations carried out with the IRAM Plateau de Bure Interferometer. IRAM is supported by INSU/CNRS (France), MPG (Germany), and IGN (Spain).

$\star \star$ Appendices are available in electronic form at

http://www . aanda.org

$\star \star \star$ The interferometrid spectra of Fig. A.1 is only available at the CDS via anonymous ftp to cdsarc.u-strasbg. fr $(130.79 .128 .5)$ or via http://cdsarc.u-strasbg.fr/viz-bin/qcat?]/A+A/568/A65
Intermediate-mass young stellar objects (IMs) are the precursors of stars in the 2-8 $M_{\odot}$ mass range (Herbig Ae/Be stars). They share some characteristics with massive young stellar objects (clustering, photodissociation regions), but they are more common than the more massive stars and therefore they can be found closer to the Sun $(d<1 \mathrm{kpc})$, which allows determination of the physical and chemical structure of their envelopes 
at similar spatial scales as can be done for low-mass protostars. From a chemical point of view, IMs are interesting because they constitute the link between the low-mass and high-mass ranges, covering an intermediate range of luminosities, densities, and temperatures.

Hot molecular cores are compact $(<0.05 \mathrm{pc})$ objects with high temperatures $(>100 \mathrm{~K})$ and densities $\left(n\left(\mathrm{H}_{2}\right)>10^{6} \mathrm{~cm}^{-3}\right)$ and they are characterized by a very rich chemistry of complex organic molecules (COMs). The typical massive hot core is the one associated with Orion KL; it hosts one of the richest molecular chemistries observed in the interstellar medium (Blake et al. 1987; Beuther et al. 2005, 2006; Tercero et al. 2010, 2011, 2013; Motiyenko et al. 2012; Cernicharo et al. 2013); COMs have also been detected coming from the inner regions of a few low-mass protostars, the so-called hot corinos (IRAS 16293-2422: Cazaux et al. 2003; Bottinelli et al. 2004a; Pineda et al. 2012; NGC 1333 IRAS 2A: Jørgensen et al. 2005, 2007; NGC1333-IRAS 4AB: Bottinelli et al. 2004b, 2007). These regions have smaller sizes $(\sim 150 \mathrm{AU})$ and lower temperatures $(\sim 100 \mathrm{~K})$. Although high sensitivity interferometric data on hot cores and corinos are still scarce and the comparison is difficult, there is some general consensus that hot corinos are richer in O-bearing molecules like $\mathrm{CH}_{3} \mathrm{OCHO}, \mathrm{CH}_{3} \mathrm{CHO}, \mathrm{CH}_{3} \mathrm{OCH}_{3}$, or $\mathrm{HCOOH}$, and poorer in $\mathrm{N}$-bearing compounds. Furthermore, large abundances of deuterated molecules and large deuterium fractions are only associated with hot corinos (Vastel et al. 2003; Parise et al. 2004; Demyk et al. 2010).

A handful of well studied hot cores exist in the IM range: NGC 7129 FIRS 2 (Fuente et al. 2005a, hereafter FU05), IC 1396 N (Neri et al. 2007; Fuente et al. 2009), IRAS 22198+6336 (Sánchez-Monge et al. 2010; Palau et al. 2011), and AFGL 5142 (Palau et al. 2011). The sizes of these IM hot cores range from $\sim 130$ AU (IRAS 22198) to $900 \mathrm{AU}$ (NGC 7129) and their chemistries present some differences. Fuente et al. $(2005 \mathrm{a}, \mathrm{b})$ proposed that IM hot cores, like hot corinos, are richer in $\mathrm{H}_{2} \mathrm{CO}$ and $\mathrm{HCOOH}$ relative to $\mathrm{CH}_{3} \mathrm{OH}$ than massive hot cores, and they did not find any variation in the $\mathrm{CH}_{3} \mathrm{CN} / \mathrm{CH}_{3} \mathrm{OH}$ abundance ratio across the stellar range. Palau et al. (2011) studied the chemistry of IRAS 22198+6336 and AFGL 5142 and concluded that these IM hot cores are richer in oxygenated molecules with two or more $\mathrm{CH}_{2 / 3}$ groups than hot corinos, but poorer in N-bearing molecules than massive hot cores. As yet, the number of studied objects is very low and the derived column densities have large uncertainties.

In this paper, we present a $4 \mathrm{GHz}$ interferometric frequency survey towards the hot core NGC 7129 FIRS 2 (hereafter, FIRS 2) that, with a luminosity of $\sim 500 L_{\odot}$, lies near the middle of the IM luminosity range. FIRS 2 is a prototypical young IM protostar; it is associated with energetic outflows (Fuente et al. 2001) and presents clear signs of CO depletion and enhanced deuterium fractionation when observed with singledish telescopes (scales of 10000 AU; Fuente et al. 2005b; Alonso-Albi et al. 2010). It is located at a distance of $1250 \pm$ 50 pc from the Sun (Shevchenko \& Yakubov Shevchenko1989) and FU05 detected a compact hot core at its center. FIRS 2 was observed with Herschel within the Water In Star-forming regions (WISH) key program (van Dishoeck et al. 2011) and it is one of the best known IM protostars with a huge set of data at both millimeter and far-IR wavelengths (Johnstone et al. 2010; Fich et al. 2010; Fuente et al. 2012). This source and IRAS $22198+6336$ are the only hot cores without clear signs of clustering which suggests that their luminosity could come from a single binary system (Palau et al. 2013).

\section{Observations}

The interferometric observations were carried out with the Plateau de Bure Interferometer (PdBI) in its CD configuration during August and November 2012. This configuration provided an angular resolution of $1.43^{\prime \prime} \times 1.26^{\prime \prime} \mathrm{PA} 144^{\circ}(\sim 1870 \mathrm{AU}$ $\times 1647 \mathrm{AU}$ at the distance of FIRS 2) at the central frequency. The $1 \mathrm{~mm}$ receivers were tuned at $219.360 \mathrm{GHz}$ which allowed the simultaneous imaging of the $\mathrm{C}^{18} \mathrm{O} 2 \rightarrow 1$ and ${ }^{13} \mathrm{CO} 2 \rightarrow 1$ lines, both within the $\sim 4 \mathrm{GHz}$ receiver's band. The narrow $40 \mathrm{MHz}$ correlator units were used to sample the $\mathrm{C}^{18} \mathrm{O}$ and ${ }^{13} \mathrm{CO}$ lines at the high spectral resolution of $40 \mathrm{kHz}$. The wideband correlator WideX sampled the whole $4 \mathrm{GHz}$ bandwidth with a spectral resolution of $2 \mathrm{MHz}$. MWC 349 was used as flux calibrator (1.92 Jy) and the rms in each WideX channel, 4-6 mJy/beam ( 0.058-0.087 K).

The interferometric observations were complemented with single-dish observations using the $30 \mathrm{~m}$ telescope and the same frequency setup. These observations were done in December 2012 and the achieved $\mathrm{rms}\left(T_{\mathrm{a}}^{*}\right)$ was $\sim 0.012 \mathrm{~K}$ (0.09 Jy) in a channel of $\sim 1.953 \mathrm{MHz}$. Therefore, the $30 \mathrm{~m} \mathrm{ob}-$ servations are $\sim 15$ times less sensitive than the $\mathrm{PdB}$ images. The telescope was pointed towards the phase center of the interferometric observations: $21^{\mathrm{h}} 43^{\mathrm{m}} 01^{\mathrm{s}} .7,+66^{\circ} 03^{\prime} 23^{\prime \prime} .6(\mathrm{~J} 2000)$. Forward and main beam efficiencies are 0.92 and 0.63 , respectively. The selected intensity scale is main beam temperature.

This paper is dedicated to the analysis of the chemical complexity revealed by the WideX data towards the mm continuum source.

\section{Results}

\subsection{Continuum maps}

The high density of lines detected made it impossible to accurately measure the continuum flux from the low spectral resolution WideX data. Instead, we used the empty emission channels around the $\mathrm{C}^{18} \mathrm{O}$ and ${ }^{13} \mathrm{CO}$ lines measured at higher spectral resolution using the $40 \mathrm{MHz}$ correlator units to determine the continuum flux. As expected, the continuum flux was slightly different at $219.560 \mathrm{GHz}$ and $220.398 \mathrm{GHz}$. We derived two continuum maps at $219.560 \mathrm{GHz}$ and $220.299 \mathrm{GHz}$. Fitting the visibilities we obtained the position of the compact source: $21^{\mathrm{h}} 43^{\mathrm{m}} 01^{\mathrm{s}} .67,+66^{\circ} 03^{\prime} 23^{\prime \prime} .7$, i.e., offset by $-0.17^{\prime \prime}$ in right ascension from our phase center. The measured fluxes are $0.37(0.01) \mathrm{Jy}$ at $219.560 \mathrm{GHz}$ and $0.38(0.01)$ at $220.398 \mathrm{GHz}$, respectively. In FU05, we determined a mm emission spectral index of 2.56 based on the continuum images at $86 \mathrm{GHz}$ and $230 \mathrm{GHz}$. The new fluxes at $219.6 \mathrm{GHz}$ and $220.4 \mathrm{GHz}$ are consistent with these results taking into account the uncertainty of $10 \%$ in the absolute flux calibration.

\subsection{Molecular lines}

In Fig. A.1 we show the WideX spectra towards the continuum peak. The continuum image at $219.560 \mathrm{GHz}$ was subtracted from the spectral maps before the cleaning process. This subtraction is not perfect since the continuum presents a smooth slope across the observed bandwidth, but it is good enough for our current detection goals.

The spectrum towards the compact source is crowded with lines, typical of those found in massive hot cores. In order to estimate the flux that the interferometer is missing, we compared the PdBI spectrum with that obtained with the $30 \mathrm{~m}$ telescope. 
Table $1.30 \mathrm{~m} / \mathrm{PdBI}$ lines.

\begin{tabular}{lccccc}
\hline \hline Line & $18 \rightarrow 17$ & $\begin{array}{c}\text { Freq. } \\
(\mathrm{MHz})\end{array}$ & $\begin{array}{c}30 \mathrm{~m} \mathrm{flux}^{1} \\
\left(\mathrm{Jy} \times \mathrm{km} \mathrm{s}^{-1}\right)\end{array}$ & $\begin{array}{c}\text { PdBI flux } \\
\text { Flux }\left(\mathrm{Jy} / \mathrm{beam} \times \mathrm{km} \mathrm{s}^{-1}\right)\end{array}$ & PdBI/30 m \\
\hline $\mathrm{O}^{13} \mathrm{CS}$ & $3_{0,3} \rightarrow 2_{0,2}$ & 218222 & $41.11(1.13)$ & $0.63(0.04)$ & $\sim 60 \%$ \\
$\mathrm{H}_{2} \mathrm{CO}$ & $24 \rightarrow 23$ & 218325 & $2.40(0.38)$ & $1.51(0.19)$ & $\sim 10 \%$ \\
$\mathrm{HC}_{3} \mathrm{~N}$ & $2_{2,0} \rightarrow 3_{1,0}$ & 218440 & $12.54(0.49)$ & $2.80(0.34)$ & $\sim 100 \%$ \\
$\mathrm{CH}_{3} \mathrm{OH}$ & $3_{2,2} \rightarrow 2_{2,1}$ & 218476 & $10.80(0.42)$ & $3.26(0.56)$ & $\sim 20 \%$ \\
$\mathrm{H}_{2} \mathrm{CO}$ & $3_{2,1} \rightarrow 2_{2,0}$ & 218760 & $10.54(0.42)$ & $2.98(0.11)$ & $\sim 30 \%$ \\
$\mathrm{H}_{2} \mathrm{CO}$ & $18 \rightarrow 17$ & 218903 & $3.11(0.44)$ & $3.29(0.05)$ & $\sim 100 \%$ \\
$\mathrm{OCS}$ & $2 \rightarrow 1$ & 219560 & $32.85(0.20)$ & $2.47(0.10)$ & $<10 \%$ \\
$\mathrm{C}^{18} \mathrm{O}$ & $10_{3,8} \rightarrow 3,7^{*}$ & 219657 & $<1.13$ & $1.04(0.11)$ & $\sim 100 \%$ \\
$\mathrm{HNCO}$ & $10_{3,7} \rightarrow 3_{3,6}{ }^{*}$ & & & & \\
& $10_{2,9} \rightarrow 9_{2,8}{ }^{*}$ & 219734 & $1.91(0.53)$ & $2.13(0.12)$ & $\sim 100 \%$ \\
$\mathrm{HNCO}$ & $10_{2,8} \rightarrow 9_{2,7}{ }^{*}$ & 219737 & & & \\
& $10_{0,1} \rightarrow 9_{0,9}$ & 219798 & $3.14(0.42)$ & $2.12(0.12)$ & $\sim 70 \%$ \\
$\mathrm{HNCO}$ & $3_{1,2} \rightarrow 2_{1,1}$ & 219909 & $1.96(0.37)$ & $1.18(0.11)$ & $\sim 60 \%$ \\
$\mathrm{H}_{2}{ }^{13} \mathrm{CO}$ & $5_{6} \rightarrow 4_{5}$ & 219949 & $43.20(2.75)$ & $8.65(0.21)$ & $\sim 20 \%$ \\
$\mathrm{SO}$ & $8_{0} \rightarrow 7_{1}$ & 220078 & $3.59(0.95)$ & $3.95(0.14)$ & $\sim 100 \%$ \\
$\mathrm{E}-\mathrm{CH}_{3} \mathrm{OH}$ & $2 \rightarrow 1$ & 220399 & $144.49(1.67)$ & $5.54(0.13)$ & $<1 \%$ \\
${ }^{3} \mathrm{CO}$ & $12_{5} \rightarrow 11_{5}$ & 220641 & $2.18(0.64)$ & $2.50(0.13)$ & $\sim 100 \%$ \\
$\mathrm{CH}_{3} \mathrm{CN}$ & $12_{4} \rightarrow 11_{4}$ & 220679 & $2.00(0.25)$ & $2.20(0.08)$ & $\sim 100 \%$ \\
$\mathrm{CH}_{3} \mathrm{CN}$ & $12_{3} \rightarrow 11_{3}$ & 220709 & $1.68(0.65)$ & $2.16(0.70)$ & $\sim 100 \%$ \\
$\mathrm{CH}_{3} \mathrm{CN}$ & $12_{2} \rightarrow 11_{2}$ & 220730 & $2.07(0.58)$ & $2.05(0.46)$ & $\sim 100 \%$ \\
$\mathrm{CH}_{3} \mathrm{CN}$ & $12_{0} \rightarrow 11_{0}{ }^{*}$ & 220747 & $5.14(0.71)$ & $4.05(0.52)$ & $\sim 100 \%$ \\
$\mathrm{CH}_{3} \mathrm{CN}$ & $12_{1} \rightarrow 11_{1}{ }^{*}$ & 220743 & & & $\sim 100 \%$ \\
\hline
\end{tabular}

Notes. Comparison between the flux measured with the $30 \mathrm{~m}$ and PdB telescopes for all the lines detected with the $30 \mathrm{~m}$ single-dish telescope. ${ }^{(1)}$ Velocity-integrated flux density in the $30 \mathrm{~m}$ spectrum. ${ }^{(2)}$ Velocity-integrated flux density towards the emission peak. ${ }^{(*)}$ Blended lines.

In Table 1 we show the list of all the lines detected with both telescopes and the fraction of flux recovered using the PdBI. We note that only the $\mathrm{H}_{2} \mathrm{CO}, \mathrm{CH}_{3} \mathrm{OH}, \mathrm{SO}, \mathrm{C}^{18} \mathrm{O}$, and ${ }^{13} \mathrm{CO}$ lines are missing a significant fraction of their fluxes when observed with the PdBI. The emission of $\mathrm{OCS}, \mathrm{HNCO}, \mathrm{CH}_{3} \mathrm{CN}$, and $\mathrm{HC}_{3} \mathrm{~N}$ comes mainly from the hot core although $20 \%$ of the flux of the lowest energy HNCO line is missing. This is also consistent with the spatial distribution as observed with the PdBI. In Fig. 1, we show the integrated intensity images of some of the more intense lines. Only ${ }^{13} \mathrm{CO}, \mathrm{C}^{18} \mathrm{O}$, and $\mathrm{SO}$ present large-scale structure. In all the other molecules the emission is point-like at the angular resolution of our observations.

\section{Line identification and rotational diagrams}

For line identification we used José Cernicharo's personal catalogue which is included in the radiative transfer and molecular excitation code MADEX (Cernicharo 2012), the JPL (Pickett 1991, 1998), and the CDMS (Müller et al. 2001, 2005) catalogues. Our procedure can be summarized as follows. We started with the identification of the lines of the most common species. The rotation temperatures, molecular column densities, and source sizes were estimated by fitting the line intensities of the whole family of molecules (all isotopologues) within the program MADEX. In these calculations, we assumed local thermodynamic equilibrium, $v_{\mathrm{LSR}}=-10 \mathrm{~km} \mathrm{~s}^{-1}$, a linewidth of $8 \mathrm{~km} \mathrm{~s}^{-1}$ and a face-on disk as the source morphology. In the case of a good fit, we created a synthesized spectrum using the derived parameters and subtracted it from the observations. Then, we went on with line identification in the residual spectrum. Sometimes, especially for the weak lines, the fit was not good enough. In these cases, we did not subtract the synthesized spectrum in order to avoid spurious features and just went on identifying the features in the original spectrum.
The number of parameters that can be determined for each species depends on the number of lines detected. For example, the source size can only be determined when the lines of the main isotopologue are optically thick. In the optically thick case, $T_{\mathrm{B}} \approx \eta_{\mathrm{ff}} \times J_{v}\left(T_{\text {rot }}\right)$, where $\eta_{\mathrm{ff}}$ is the beam filling factor, and $J_{v}\left(T_{\text {rot }}\right)=h v / k T_{\text {rot }} \times\left(1-\exp \left(h v / k T_{\text {rot }}\right)\right)^{-1}$ with $T_{\text {rot }}$ the rotation temperature. Provided that we know $T_{\text {rot }}$, we can estimate the effective size of the emitting region from the line intensities. The emission sizes cannot be constrained if the main isotopologue lines are optically thin.

For the less abundant molecules, the number of detected lines was too low or the line intensities were too uncertain to estimate the rotation temperature. In these cases, a rotation temperature of $\sim 200 \mathrm{~K}$ was assumed and only the molecular column densities are calculated using MADEX. In Sects. 4.1 to 4.19, we give a more detailed description of the fitting for each species and the results of these calculations are shown in Tables 2 and 3.

Frequencies and line identifications are shown in Table A.1. We compare our synthesized spectrum including all species with observations in Fig. A.1.

\subsection{Methyl cyanide: $\mathrm{CH}_{3} \mathrm{CN}, \mathrm{CH}_{3} \mathrm{NC}$}

Methyl cyanide $\left(\mathrm{CH}_{3} \mathrm{CN}\right)$ is one of the best thermometers in hot cores. The symmetric rotor presents a $K$-ladder structure with transitions that are easily thermalized at the densities prevailing in hot cores. In addition, it is well known that this molecule is especially abundant in these regions. We have detected the whole $12_{K} \rightarrow 11_{K}$ ladder, from $K=0$ to $K=11$ in the $v=0$ ground vibrational state and in the $v_{8}=1$ vibrational level at $E_{\text {vib }}=525.17 \mathrm{~K}$ (see Table A.4). The high number of detected lines would allow us to determine the rotation and vibrational temperature of this molecule providing that the emission is optically thin (see Fig. 2). As discussed below, this is not the case 


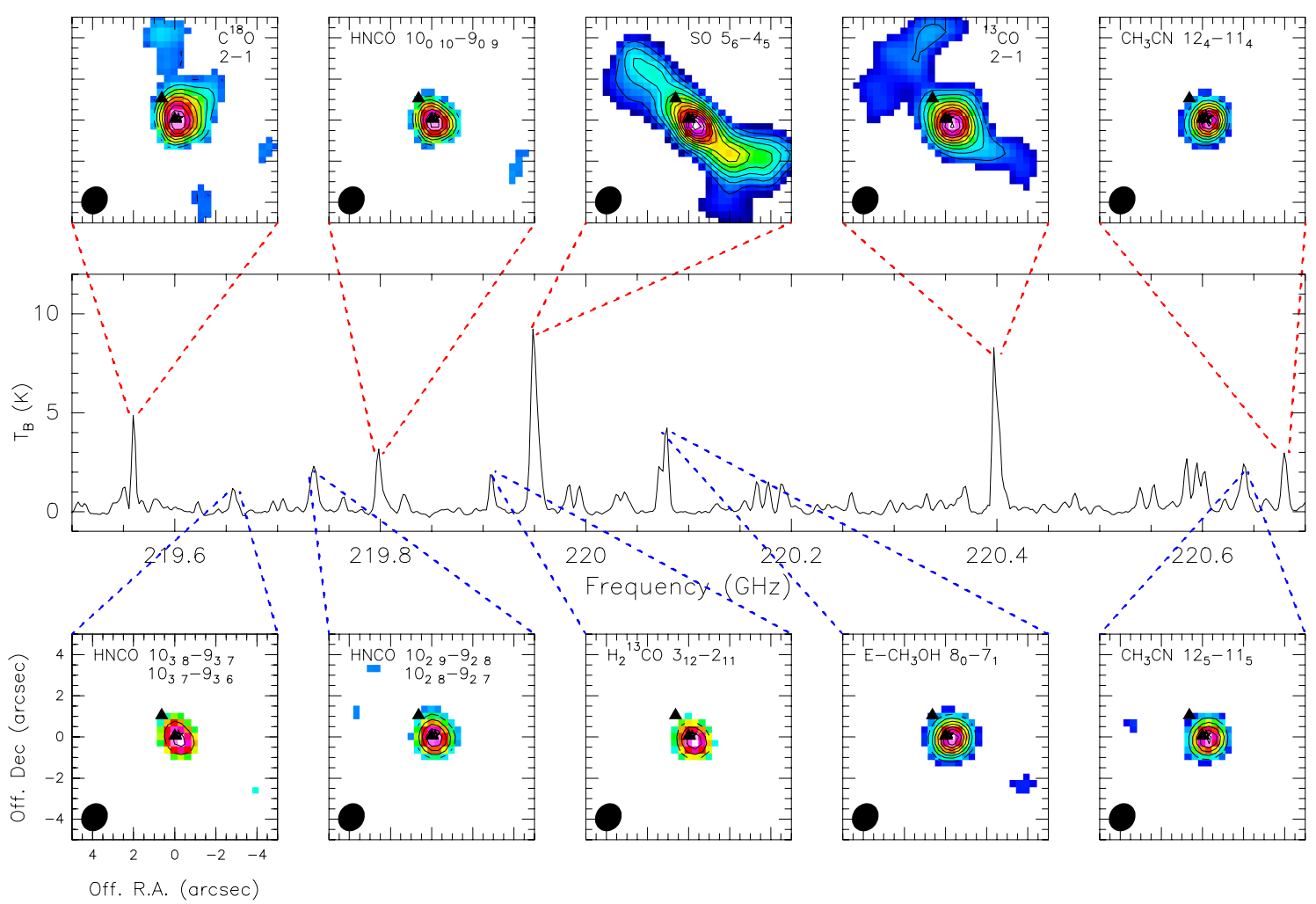

Fig. 1. Middle: PdBI spectrum towards the emission peak. Top and bottom: integrated intensity maps of the most intense lines in the portion of spectrum between $219.5 \mathrm{GHz}$ and $220.7 \mathrm{GHz}$. In all the panels the contours start and increases at steps of $3 \times \sigma$. Sigma and peak values in Jy/beam $\times \mathrm{km} \mathrm{s}^{-1}$ are: $0.10,2.47\left(\mathrm{C}^{18} \mathrm{O} 2-1\right) ; 0.12,2.12\left(\mathrm{HNCO} 10_{0}-9_{0}\right) ; 0.21,8.65\left(\mathrm{SO}_{6}-4_{5}\right) ; 0.22,7.28\left({ }^{13} \mathrm{CO} 2-1\right) ; 0.08,2.20\left(\mathrm{CH}_{3} \mathrm{CN}_{12}-11_{4}\right)$; 0.11, $1.04\left(\mathrm{HNCO} \mathrm{10}{ }_{3}-9_{3}\right) ; 0.12,2.13\left(\mathrm{HNCO} \mathrm{10}{ }_{2}-9_{2}\right) ; 0.11,1.18\left(\mathrm{H}_{2}^{13} \mathrm{CO}_{1,2}-2_{1,1}\right) ; 0.14,3.95\left(\mathrm{E}_{-} \mathrm{CH}_{3} \mathrm{OH} 8_{0}-7_{1}\right) ; 0.13,2.5\left(\mathrm{CH}_{3} \mathrm{CN}_{12}-11_{5}\right)$. Filled triangles indicate the water masers as observed with the NRAO Very Large Array (Aina Palau, priv. comm.). The masers trace the direction of the youngest outflow (Fuente et al. 2001) that is also clearly seen in the SO and ${ }^{13} \mathrm{CO}$ images. The $(0,0)$ position is the phase center of the interferometric observations: $21^{\mathrm{h}} 43^{\mathrm{m}} 01^{\mathrm{s}} .7,+66^{\circ} 03^{\prime} 23^{\prime \prime} 6(\mathrm{~J} 2000)$.

Table 2. Results of the rotational diagram analysis.

\begin{tabular}{lccc}
\hline \hline Species & $T_{\text {rot }}(\mathrm{K})$ & $N\left(\mathrm{~cm}^{-2}\right)$ & $R^{1}$ \\
\hline $\mathrm{HNCO}$ & $260_{-5}^{+4}$ & $1.6_{-0.1}^{+0.1} \times 10^{15}$ & 0.99 \\
$\mathrm{CH}_{3} \mathrm{CN} v=0^{2}$ & $905_{-161}^{+250}$ & $1.6_{-0.2}^{+0.2} \times 10^{15}$ & 0.90 \\
$\mathrm{CH}_{3} \mathrm{CN} v_{8}=1$ & $405_{-67}^{+100}$ & $9.4_{-3.1}^{+4.6} \times 10^{14}$ & 0.81 \\
$\mathrm{CH}_{3} \mathrm{OCHO} v_{t}=0,1$ & $265_{-69}^{+139}$ & $2.8_{-0.8}^{+1.9} \times 10^{16}$ & 0.60 \\
$\mathrm{CH}_{2} \mathrm{DOH}$ & $157_{-13}^{+15}$ & $1.4_{-0.3}^{+0.3} \times 10^{16}$ & 0.99 \\
$\mathrm{aGg}{ }^{-}\left(\mathrm{CH}_{2} \mathrm{OH}\right)_{2}$ & $145_{-25}^{+37}$ & $2.0_{-0.3}^{+0.3} \times 10^{15}$ & 0.92 \\
\hline
\end{tabular}

Notes. Beam filling solution. ${ }^{(1)}$ Correlation coefficient of the least squares fitting. ${ }^{(2)}$ This fit was not used in the model because the detection of the ${ }^{13} \mathrm{C}$ isotopologue showed that the lines are optically thick.

of the $v=0$ lines. Instead, we used the $v_{8}=1$ lines to estimate the rotation temperature. Unfortunately, most of the lines are blended or partially blended. This is the cause of the large uncertainties in the integrated intensities shown in Table A.4. All the lines have $T_{\mathrm{b}}>3 \sigma$, but the uncertainty in the linewidth is large for partially blended lines. As a first step we plotted all the detected lines in a rotational diagram and visually checked if any of them were clearly above or below the straight line defined by the other points. We removed the line at $221265 \mathrm{MHz}$ that was clearly above the fit and derived $T_{\text {rot }}=405_{-67}^{+100} \mathrm{~K}$ using the rest of the points (the parameters of this fit are shown in Table 2). The fit was good $(R=0.81)$ suggesting that the contribution of other species to the measured line integrated intensities were within the errors. To corroborate this assumption, as a second step, we removed the lines that were severely blended (difference in frequency less than $4 \mathrm{MHz}$ ) and kept only those that were isolated or partially blended. In the end we had only eight transitions and derived $T_{\text {rot }}=609_{-167}^{+357} \mathrm{~K}$. The higher rotation temperature was due to the suppression of the lines at $221199,221394,221403$, and $221422 \mathrm{MHz}$. The obtained value was still in agreement with our previous fit within the errors. We were worried because the velocity integrated line intensities of the transitions with $E_{\text {rot }}>400 \mathrm{~K}(221059,221210 \mathrm{MHz})$ present large uncertainties. In order to check the robustness of our temperature estimate, we make a new least squares fitting without these lines. This fit provided a lower limit to the rotation temperature of $T_{\text {rot }}=250_{-59}^{+110} \mathrm{~K}$. Again the value obtained for the rotation temperature is in agreement with that shown in Table 2 within the errors. Therefore, we decided to keep the estimate shown in Table 2 as a good value for the average rotation temperature, although we are aware that, more likely, we have strong temperature gradients across the hot core with temperatures varying between $\sim 250 \mathrm{~K}$ and $\sim 600 \mathrm{~K}$.

The results from the $\mathrm{CH}_{3} \mathrm{CN} v=0$ rotational diagram are shown in Table 2. We obtained a higher rotational temperature in $v=0$ than in $v_{8}=1$. One could think that the high temperature in the ground vibrational state was an artifact due to the missing flux in the low $K$ components of the $\mathrm{CH}_{3} \mathrm{CN} 12_{K} \rightarrow 11_{K}$ ladder. However, comparison of the interferometric PdBI and single-dish $30 \mathrm{~m}$ observations showed that the spatial filtering is not important and therefore this possibility was discarded. An alternative explanation is that the ground state vibrational 

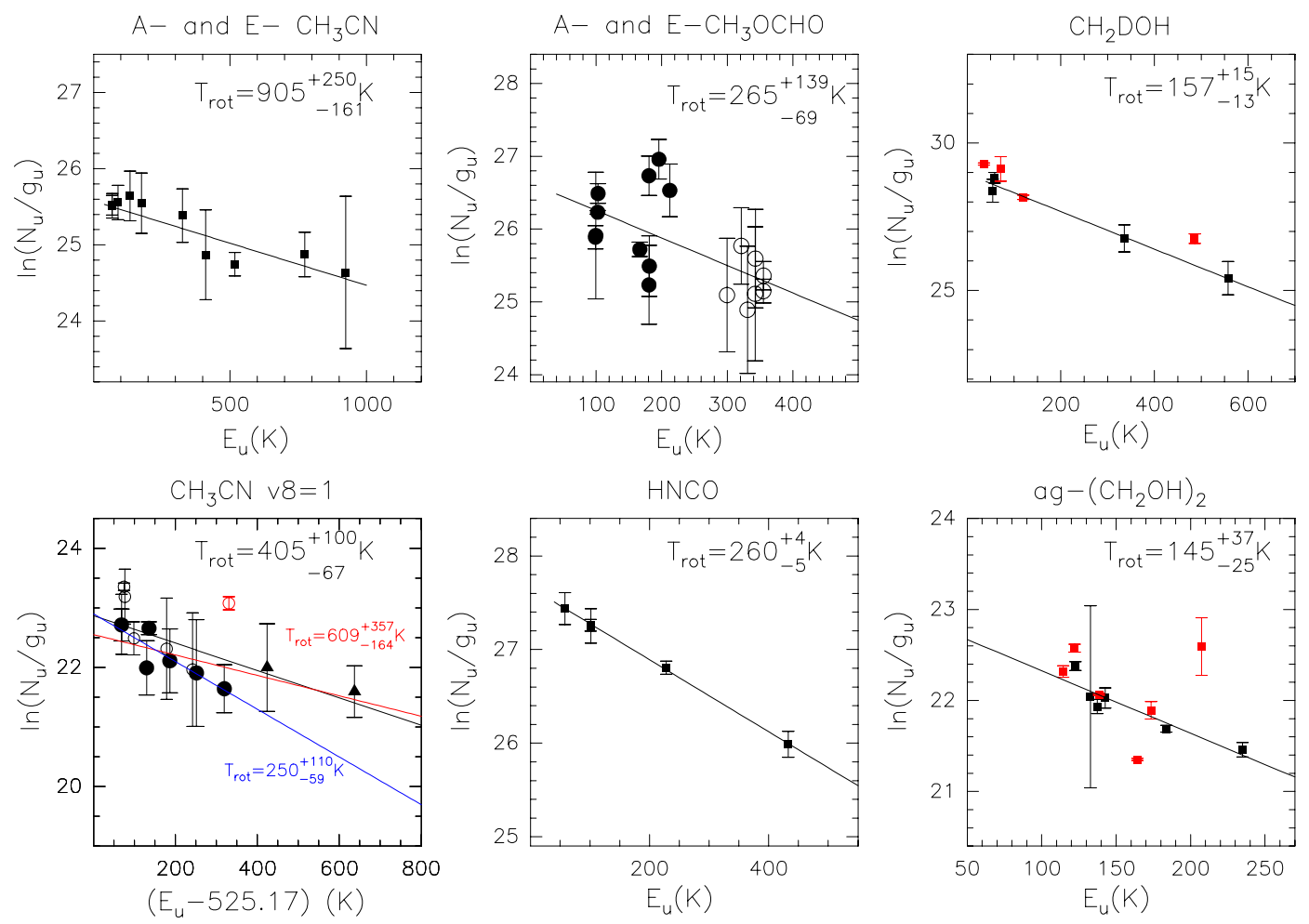

Fig. 2. Results of the rotational diagrams for $\mathrm{CH}_{3} \mathrm{CN} v=0, \mathrm{CH}_{3} \mathrm{CN} v_{8}=1, \mathrm{CH}_{3} \mathrm{OCHO}, \mathrm{HNCO}, \mathrm{CH}_{2} \mathrm{DOH}$ and aGg'- $\left(\mathrm{CH}_{2} \mathrm{OH}\right)_{2}$. The high rotation temperature fitted to the $\mathrm{CH}_{3} \mathrm{CN} v=0$ rotational diagram is the consequence of the lines being optically thick and is not considered in our modeling (see Sect. 4.1). In the rotational diagram of the $\mathrm{CH}_{3} \mathrm{CN} v_{8}=1$ vibrational state we use different symbols for: blended lines (empty circles), isolated or partially blended lines (filled circles), and the points with $E_{\mathrm{rot}}>400 \mathrm{~K}$ (triangles). The three straight lines correspond to the least squares fittings with all the points (black), only unblended lines (red), and unblended lines with $E_{\mathrm{rot}}<400 \mathrm{~K}$ (blue). For $\mathrm{CH}_{3} \mathrm{OCHO}$, the points corresponding to the $v=0$ lines are indicated with filled circles and $v_{t}=1$ with empty circles. The parameters of the transitions used in the rotation diagrams are listed in Tables A.3-A.8. Red points have not been used in the least squares fitting because they correspond to heavily blended lines.

lines are optically thick. The opacity can be derived by comparing the $\mathrm{CH}_{3} \mathrm{CN}$ and $\mathrm{CH}_{3}{ }^{13} \mathrm{CN}$ lines (the lines of the other ${ }^{13} \mathrm{C}$ isotopologue ${ }^{13} \mathrm{CH}_{3} \mathrm{CN}$ are outside the observed frequency range). Unfortunately, the $\mathrm{CH}_{3}{ }^{13} \mathrm{CN} 12_{0} \rightarrow 11_{0}$ and $12_{1} \rightarrow 11_{1}$ lines are blended with those of the main isotopologue. The lowest $K$ transition without any obvious contamination is $K=4$ $(220571 \mathrm{MHz})$ where we have a feature with $T_{\mathrm{b}} \sim 0.39 \mathrm{~K}$ (with an uncertainty of $25 \%$ because of partial blending with other lines). Comparing with the main isotopologue, we have $I\left(\mathrm{CH}_{3} \mathrm{CN} 12_{4} \rightarrow 11_{4}\right) / I\left(\mathrm{CH}_{3}{ }^{13} \mathrm{CN} 12_{4} \rightarrow 11_{4}\right) \sim 7$, which implies opacities of $\sim 10$ in the main isotopologue line. Since the line opacities are very high, the rotational diagram analysis is not valid and the resulting rotation temperature in the ground state is severely over-estimated. Instead, we derived the $\mathrm{CH}_{3} \mathrm{CN}$ column density assuming $T_{\text {rot }}=405 \mathrm{~K}$ (the same as in the $v_{8}=1$ vibrational level) and an opacity of $\sim 10$ for the $\mathrm{CH}_{3} \mathrm{CN} 12_{4} \rightarrow 11_{4}$ line and obtained $N\left(\mathrm{E}-\mathrm{CH}_{3} \mathrm{CN}\right)=N(\mathrm{~A}-$ $\left.\mathrm{CH}_{3} \mathrm{CN}\right)=2.6 \times 10^{18} \mathrm{~cm}^{-2}$, i.e., a total $(\mathrm{A}+\mathrm{E}) \mathrm{CH}_{3} \mathrm{CN}$ column density of $5.2 \times 10^{18} \mathrm{~cm}^{-2}$. With these parameters, we fit the high energy rotational lines with a source size of $\approx 0.07^{\prime \prime}$ (125 AU). The uncertainty in the source size comes from the assumed rotation temperature. By varying the rotation temperature between $250 \mathrm{~K}$ and $600 \mathrm{~K}$ we would obtain sizes of 100-160 AU. We note that these sizes are quite small, similar to typical scales of circumstellar disks.

This compact source model successfully predicted the intensities of the high excitation lines, but the intensities of the low-energy transitions were severely underestimated. The only way to fit the whole spectrum was to consider a two-component model: (i) the compact component described above; and (ii) a more extended second component that is filling the beam. We fit the $K<4$ lines with $N\left(\mathrm{E}-\mathrm{CH}_{3} \mathrm{CN}\right)=N\left(\mathrm{~A}-\mathrm{CH}_{3} \mathrm{CN}\right)=2.5 \times$ $10^{14} \mathrm{~cm}^{-2}$ and $T_{\text {rot }}=350 \mathrm{~K}$ for this extended component. We tried with a temperature of $250 \mathrm{~K}$ obtained from the fitting of the $E_{\text {rot }}<400 \mathrm{~K} v_{8}=1$ lines, but this lower temperature would overestimate the $K=0$ and $K=1$ lines. Furthermore, the assumed value is within the uncertaintly of this fit $\left(=250_{-59}^{+110} \mathrm{~K}\right)$.

Our estimate of the total methyl cyanide column density relies on the ${ }^{13} \mathrm{C}$ isotopologue line. Of course there is also the possibility that the $\mathrm{CH}_{3}{ }^{13} \mathrm{CN} 12_{4} \rightarrow 11_{4}$ line is contaminated by another line and its real intensity is lower. We did not find any good candidates at this frequency. One line of EA- $\left(\mathrm{CH}_{3}\right)_{2} \mathrm{CO}$ is very close in frequency, but the emission of this line is very weak (see also Sect. 4.7). Furthermore, the small sizes derived for other species (see following sections) prompted us to consider the two-component model as the most likely one. We would also like to point out that we refer to a beam filling component at the spatial resolution of our observations $(\sim 1900 \mathrm{AU})$ as "extended component". This component would not encompass the entire envelope.

We also looked for the deuterated compounds in our spectrum. The most abundant species $\mathrm{CH}_{2} \mathrm{DCN}$ and $\mathrm{CHD}_{2} \mathrm{CN}$ have no intense lines in the observed frequency range. Only $\mathrm{CD}_{3} \mathrm{CN}$ has three intense transitions at 219.965, 219.983, and $219.993 \mathrm{GHz}$. We have detected the three lines but need a very low rotation temperature, $T_{\text {rot }}=40 \mathrm{~K}$, to fit them. Because of this surprisingly low rotation temperature, we consider that a misidentification is possible and keep the derived 
column density as an upper value to the real one. Several weak lines of $\mathrm{CH}_{3} \mathrm{NC}$ were identified, a molecule so far only detected towards Sgr B2 (Cernicharo et al. 1988). Unfortunately, most of them are blended or partially blended. Assuming that $\mathrm{CH}_{3} \mathrm{NC}$ is coming from the $\mathrm{CH}_{3} \mathrm{CN}$ compact core, we calculated a $\mathrm{CH}_{3} \mathrm{NC}$ column density of $1.5 \times 10^{16} \mathrm{~cm}^{-2}$, i.e., about 350 times lower than that of $\mathrm{CH}_{3} \mathrm{CN}$. Since we have only one unblended line, we keep this detection as tentative.

\subsection{Isocyanic acid: $H N C O, H N^{13} C O$}

The HNCO lines are well fitted with $T_{\text {rot }}=260_{-5}^{+4} \mathrm{~K}$ and $N(\mathrm{HNCO})=1.6 \times 10^{15} \mathrm{~cm}^{-2}$ (see Fig. 2 and Table 2). In our frequency range, most of the lines of $\mathrm{HN}^{13} \mathrm{CO}$ are blended with those of the main isotopologue and so we cannot estimate the line opacities. Comparing this with the $30 \mathrm{~m}$ spectrum, we calculated that the interferometer is missing about $20 \%$ of the flux of the low energy lines, showing that there is an extended component. For this reason, we adopt the beam filling solution. We looked for the isomer HCNO and the deuterated compound DNCO, but unfortunately they do not have intense transitions in the sampled frequency range.

\subsection{Methyl formate: $\mathrm{A}-\mathrm{CH}_{3} \mathrm{OCHO}, \mathrm{E}-\mathrm{CH}_{3} \mathrm{OCHO}$}

We detected more than sixty lines of $\mathrm{A}-\mathrm{CH}_{3} \mathrm{OCHO}$ and $\mathrm{E}-\mathrm{CH}_{3} \mathrm{OCHO}$ in our spectrum, both in the $v_{t}=0,1$ vibrational states, the latter at $E_{\text {vib }}=188.40 \mathrm{~K}$. We tried to fit a rotational diagram to each vibrational state separately in order to measure the rotation and vibrational temperatures independently, but this was not possible. The dynamical range of $E_{u}$ within each vibrational level is very small and the uncertainties in the integrated intensities are too large to obtain a good fit. However, we could get a reasonable fit by considering the two vibrational levels together (see Table 2 ). We measured $T_{\text {vib,rot }} \sim 265_{-69}^{+139} \mathrm{~K}$, slightly lower than that of $\mathrm{CH}_{3} \mathrm{CN}$, but still in good agreement taking into account the associated uncertainties. We fixed the temperature and varied the source size to fit the lines. The best fit was obtained with a size of $\sim 0.1^{\prime \prime}$. Even with this small size, the opacities of the $\mathrm{CH}_{3} \mathrm{OCHO} v=0$ lines are always $\leq 1.3$ suggesting that our estimate of the rotation temperature is reliable. Unfortunately, the lines of the ${ }^{13} \mathrm{C}$ isotopologues are blended with other species and it was not possible to have an estimate of their column densities. We used a weak feature at $128.952 \mathrm{GHz}$ to derive an upper limit to the total column density of ${ }^{13} \mathrm{CH}_{3} \mathrm{OCHO}$ of $<3.0 \times 10^{17} \mathrm{~cm}^{-2}$ in the $\sim 0.1^{\prime \prime}$ core, which would imply an upper limit to the total (A- $+\mathrm{E}-)$ main isotopologue column density of $<2.0 \times 10^{19} \mathrm{~cm}^{-2}$ assuming ${ }^{12} \mathrm{C} /{ }^{13} \mathrm{C}=65$. This allows us to say that the methyl formate column density is accurate within a factor of 4 . When considering deuteration, we have three detected lines at $219242 \mathrm{MHz}, 218730 \mathrm{MHz}$, and $219132 \mathrm{MHz}$ that would correspond to $\mathrm{CH}_{3} \mathrm{OCDO}$ and $\mathrm{CH}_{2}$ DOCHO. The first two lines are blended with lines of EE- $\left(\mathrm{CH}_{3}\right)_{2} \mathrm{CO}$ which makes the derived deuterium fraction more uncertain. Taking into account the contribution of acetone, we fitted the intensities of the three lines assuming a deuterium fraction of $\sim 0.06$.

\subsection{Ethyl cyanide: $\mathrm{CH}_{3} \mathrm{CH}_{2} \mathrm{CN}$}

The detection of eight lines of $\mathrm{CH}_{3} \mathrm{CH}_{2} \mathrm{CN}$ strongly confirms the identification of this species. Although three of them are heavily blended, the other five lines are sufficient to identify this species. The lines belong to the ground state and to the lowest energy vibrational states at $E_{\text {vib }}=297.12 \mathrm{~K}$ (Daly et al. 2013). We tried to fit the rotational diagram, but unfortunately the points did not follow a clear pattern and we could not determine the rotation temperature. Hence, we fixed the rotation temperature to a value of $200 \mathrm{~K}$ (a reasonable value from the rotational temperatures measured with the other species; see Table 2) and fitted the column density. A beam averaged column density value of $5.2 \times 10^{14} \mathrm{~cm}^{-2}$ was derived from the intensity of the most intense lines. The fit improved assuming that the emission comes from a compact source with a diameter of $\sim 0.1^{\prime \prime}$, because some lines become optically thick. This is the solution we finally adopted (see Table 3 ). We searched for the lines of the ${ }^{13} \mathrm{C}$ isotopologues and the deuterated species, but the lines were too weak to obtain a significant upper limit to their column densities.

\subsection{Methanol: A- and E- $\mathrm{CH}_{3} \mathrm{OH}, \mathrm{A}$ - and $\mathrm{E}-{ }^{13} \mathrm{CH}_{3} \mathrm{OH}$, $\mathrm{CH}_{2} \mathrm{DOH}$}

Only five lines of methanol (including the A- and E- species and their vibrational states) were detected and their intensities were not consistent with LTE. This is not unexpected since the emission of the low energy lines is optically thick and extended (see Table 1). We searched for the lines of ${ }^{13} \mathrm{CH}_{3} \mathrm{OH}$ and the deuterated species $\mathrm{CH}_{2} \mathrm{DOH}$. Ten lines of $\mathrm{CH}_{2} \mathrm{DOH}$ were detected. We removed from our fit the lines that could be blended with other more intense lines and we still kept four clean lines that allowed us to derive the results shown in Fig. 2 and Table 2. The rotation temperature of $\mathrm{CH}_{2} \mathrm{DOH}, \sim 157_{-13}^{+15} \mathrm{~K}$ is lower than that of $\mathrm{CH}_{3} \mathrm{CN}$, suggesting that it arises from a different region within the hot core. Based on the different spatial distribution of the high excitation and low excitation lines of methanol, FU05 proposed the existence of two components: a hot component towards the hot core and a colder component that extends along the outflow direction. Although the two components are expected to contribute to the total $\mathrm{CH}_{2} \mathrm{DOH}$ emission, its low rotation temperature suggests that it is dominated by the extended one.

For ${ }^{13} \mathrm{CH}_{3} \mathrm{OH}$, we detected two lines of the $v_{t}=0$ vibrational state (220323 and $221282 \mathrm{MHz}$ ) and one (221 424 MHz) of the $v_{t}=1$ state. Although the emissions of the low energy lines of the main isotopologue are extended (see Table 1), we considered that the emissions of the 13-methanol lines come mainly from the compact source. The line at $220323 \mathrm{MHz}$ is heavily blended and cannot be used to estimate the 13-methanol column density. Using the other two lines we fitted a vibrational/rotation temperature of $\sim 238 \mathrm{~K}$ and a total ${ }^{13} \mathrm{CH}_{3} \mathrm{OH} v_{t}=0,1$ column density of $5.3 \times 10^{18} \mathrm{~cm}^{-2}$. In order to estimate the uncertainty in our estimate of the 13-methanol column density, we repeated the fit by fixing the rotation temperature to $200 \mathrm{~K}$ and $400 \mathrm{~K}$. The derived column densities are $3.0 \times 10^{18} \mathrm{~cm}^{-2}$ and $6.1 \times 10^{18} \mathrm{~cm}^{-2}$, respectively, which suggests that our estimate of the ${ }^{13} \mathrm{C}$-methanol column density is accurate within a factor of 2 .

Since the $\mathrm{CH}_{3} \mathrm{OH}$ lines are optically thick, we derived the methanol column density from ${ }^{13} \mathrm{CH}_{3} \mathrm{OH}$ assuming that all the ${ }^{13} \mathrm{CH}_{3} \mathrm{OH}$ emission comes from the compact component and ${ }^{12} \mathrm{C} /{ }^{13} \mathrm{C}=65$. Then we added an extended component to fit the rest of lines. The value obtained for the $\mathrm{CH}_{3} \mathrm{OH}$ column density in the extended component is not reliable because the intensities of the main isotopologue lines are affected by spatial filtering and opacity effects. Moreover, the separation of the methanol emission in these two components is model-dependent since we fixed the size of the compact core in our calculations. The beam average $\mathrm{CH}_{3} \mathrm{OH}$ column density derived from ${ }^{13} \mathrm{CH}_{3} \mathrm{OH}$ is, however, reliable as long as the 
A. Fuente et al.: The hot core towards the intermediate-mass protostar NGC 7129 FIRS 2

${ }^{13} \mathrm{C}$-isotopologue is optically thin regardless of the detailed spatial distribution of methanol within the observational beam. For this reason, we used this value to calculate the deuterium fraction, $\left[\mathrm{CH}_{2} \mathrm{DOH}\right] /\left[\mathrm{CH}_{3} \mathrm{OH}\right]=0.02$. This is an average value within the hot core.

\subsection{Trans- and gauche-ethanol: $\mathrm{T}-$ and $\mathrm{G}-\mathrm{CH}_{3} \mathrm{CH}_{2} \mathrm{OH}$}

We detected six ethanol lines which includes four from $\mathrm{T}-\mathrm{CH}_{3} \mathrm{CH}_{2} \mathrm{OH}$ and two from the $\mathrm{G}$ conformer that is at $60 \mathrm{~K}$ above the $\mathrm{T}$ state. However, only three lines are clearly detected (at 218 554, 218 655, and $220155 \mathrm{MHz}$ ) since the other are significantly blended with transitions from other species or $\mathrm{U}$ lines. Because of this, we could not apply the rotational diagram technique. Instead, we assumed a rotation temperature of $T_{\text {rot }}=200 \mathrm{~K}$, and derived the column densities using the most intense lines. We assumed a size of $\sim 0.1^{\prime \prime}$ to more accurately fit the data published by FU05 (see Sect. 5). The results are shown in Table 3.

\subsection{Acetone: $\mathrm{AA}-\left(\mathrm{CH}_{3}\right)_{2} \mathrm{CO}, \mathrm{AE}-\left(\mathrm{CH}_{3}\right)_{2} \mathrm{CO}, \mathrm{EA}-\left(\mathrm{CH}_{3}\right)_{2} \mathrm{CO}$, $\mathrm{EE}-\left(\mathrm{CH}_{3}\right)_{2} \mathrm{CO}$}

Several lines of acetone were detected in our spectrum (218773, 219076, 219220, 219242, 219264, 220 355, 220 466, 220764 , $220962 \mathrm{MHz}$ ), the most intense being at 219220, 219242 , and $219264 \mathrm{MHz}$. Because many of them are blended, it is not possible to determine an accurate rotation temperature. We assumed $T_{\text {rot }}=200 \mathrm{~K}$ and obtained a total $(\mathrm{AA}+\mathrm{EA}+\mathrm{AE}+\mathrm{EE})$ beam average column density of $1.0 \times 10^{15} \mathrm{~cm}^{-2}$.

\subsection{Formaldehyde: $\mathrm{H}_{2} \mathrm{CO}$}

Three lines of $\mathrm{p}-\mathrm{H}_{2} \mathrm{CO}$ and one of $\mathrm{o}-\mathrm{H}_{2}{ }^{13} \mathrm{CO}$ were detected in the PdBI spectrum. The lines of the main isotopologue are optically thick and most of their emission has been filtered by the interferometer (see Table 1). Because of the large fraction (85\%) of missing flux in the main isotopologue line, we are surely missing an extended component. As can be seen in Fig. 1, the emission of the $\mathrm{o}-\mathrm{H}_{2}{ }^{13} \mathrm{CO}$ is unresolved by our observations. In addition, FU05 derived a size of $0.58 \pm 0.24^{\prime \prime} \times 0.40 \pm 0.24^{\prime \prime}$ for the $\mathrm{D}_{2} \mathrm{CO}$ emission. Based on these results, we were able to fit all the lines of this work and FU05 with an (ortho+para) $-\mathrm{H}_{2}{ }^{13} \mathrm{CO}$ column density of $1.1 \times 10^{16} \mathrm{~cm}^{-2}$ and (ortho+para)- $\mathrm{D}_{2} \mathrm{CO}$ column density of $5.9 \times 10^{15} \mathrm{~cm}^{-2}$, and a source with a diameter of $0.34^{\prime \prime}$ ( $\sim 26 \mathrm{AU})$. We assume $T_{\text {rot }}=200 \mathrm{~K}$ and an ortho-to-para ratio of 3 .

There are no $\mathrm{H}_{2} \mathrm{C}^{18} \mathrm{O}$ and $\mathrm{HDCO}$ lines in the frequency range of our observations that we could use to further constrain our model.

\subsection{Carbonyl sulfide: OCS, $\mathrm{O}^{13} \mathrm{CS}$}

The $J=19 \rightarrow 18$ rotational lines of the ground vibrational states of OCS and $\mathrm{O}^{13} \mathrm{CS}$ lie in the range of wavelengths covered by our observations. We measured $T_{\mathrm{b}}[\mathrm{OCS} 19 \rightarrow 18] /$ $T_{\mathrm{b}}\left[\mathrm{O}^{13} \mathrm{CS} 19 \rightarrow 18\right] \sim 5$. Comparing the $30 \mathrm{~m}$ and $\mathrm{PdB}$ spectra, we discarded the possibility that this low intensity ratio is a consequence of the spatial filtering. The opacity of the OCS $19 \rightarrow 18$ line must be very high, $\tau \sim 12$. Assuming $T_{\text {rot }}=$ $200 \mathrm{~K}$, we derived a OCS column density of $\sim 2 \times 10^{18} \mathrm{~cm}^{-2}$ and a source size of $\approx 0.32^{\prime \prime}$ (400 AU), four times that derived for the compact component of $\mathrm{CH}_{3} \mathrm{CN}$. In our spectrum, we
Table 3. NGC 7129 FIRS 2 hot core model.

\begin{tabular}{|c|c|c|c|c|}
\hline Species & $T_{\text {rot }}(\mathrm{K})$ & $N_{X}\left(\mathrm{~cm}^{-2}\right)^{1}$ & $D\left({ }^{\prime \prime}\right)^{2}$ & \\
\hline \multirow[t]{2}{*}{$\mathrm{CH}_{3} \mathrm{CN} v=0$} & 405 & $5.2 \times 10^{18}$ & 0.07 & $E^{4}$ \\
\hline & 350 & $5.0 \times 10^{14}$ & $\mathrm{BF}^{3}$ & $\mathrm{G}^{5}$ \\
\hline $\mathrm{CH}_{3} \mathrm{CN} v_{8}=1$ & 405 & $7.0 \times 10^{16}$ & 0.07 & $\mathrm{G}$ \\
\hline \multirow[t]{2}{*}{$\mathrm{CH}_{3}{ }^{13} \mathrm{CN} v=0$} & 405 & $8.0 \times 10^{16}$ & 0.07 & $\mathrm{E}$ \\
\hline & 350 & $7.7 \times 10^{12}$ & $\mathrm{BF}$ & $\mathrm{G}$ \\
\hline $\mathrm{CH}_{3} \mathrm{NC}^{*}$ & 405 & $3.0 \times 10^{16}$ & 0.07 & $\mathrm{G}$ \\
\hline $\mathrm{CD}_{3} \mathrm{CN}^{*}$ & 40 & $<5.0 \times 10^{13}$ & $\mathrm{BF}$ & G \\
\hline \multirow{2}{*}{$\mathrm{CH}_{3} \mathrm{OH} v_{t}=0,1$} & 238 & $3.4 \times 10^{20}$ & 0.12 & $\mathrm{G}$ \\
\hline & $157^{6}$ & $2.0 \times 10^{16}$ & $\mathrm{BF}$ & G \\
\hline${ }^{13} \mathrm{CH}_{3} \mathrm{OH} v=0$ & 238 & $4.2 \times 10^{18}$ & 0.12 & $\mathrm{G}$ \\
\hline${ }^{13} \mathrm{CH}_{3} \mathrm{OH} v_{t}=1$ & 238 & $1.1 \times 10^{18}$ & 0.12 & $\mathrm{G}$ \\
\hline $\mathrm{CH}_{2} \mathrm{DOH}$ & 157 & $1.4 \times 10^{16}$ & $\mathrm{BF}$ & $\mathrm{G}$ \\
\hline $\mathrm{CH}_{3} \mathrm{OCHO} v=0$ & 265 & $3.4 \times 10^{18}$ & 0.12 & $\mathrm{E}$ \\
\hline $\mathrm{CH}_{3} \mathrm{OCHO} v_{t}=1$ & 265 & $1.7 \times 10^{18}$ & 0.12 & $\mathrm{E}$ \\
\hline $\mathrm{CH}_{3} \mathrm{OCDO}$ & 265 & $1.6 \times 10^{17}$ & 0.12 & $\mathrm{G}$ \\
\hline $\mathrm{S}-\mathrm{CH}_{2} \mathrm{DOCHO}$ & 265 & $8.0 \times 10^{16}$ & 0.12 & $\mathrm{G}$ \\
\hline $\mathrm{A}-\mathrm{CH}_{2} \mathrm{DOCHO}$ & 265 & $8.0 \times 10^{16}$ & 0.12 & $\mathrm{G}$ \\
\hline $\mathrm{CH}_{3} \mathrm{CH}_{2} \mathrm{CN}$ & 200 & $9.4 \times 10^{16}$ & 0.12 & $\mathrm{E}$ \\
\hline $\mathrm{CH}_{3} \mathrm{CH}_{2} \mathrm{CN} v_{13}=1 / v_{21}=1$ & 200 & $2.9 \times 10^{16}$ & 0.12 & $\mathrm{E}$ \\
\hline $\mathrm{CH}_{2} \mathrm{CHCN} v_{11}=0,1$ & 200 & $<9.4 \times 10^{17}$ & 0.12 & $\mathrm{G}$ \\
\hline $\mathrm{CH}_{3} \mathrm{CH}_{2} \mathrm{OH}$ & 200 & $3.0 \times 10^{18}$ & 0.12 & $\mathrm{E}$ \\
\hline $\mathrm{CH}_{3} \mathrm{COCH}_{3}$ & 200 & $1.0 \times 10^{15}$ & $\mathrm{BF}$ & $\mathrm{G}$ \\
\hline$(\mathrm{o}+\mathrm{p})-\mathrm{H}_{2} \mathrm{CO}$ & 200 & $7.0 \times 10^{17}$ & 0.34 & $\mathrm{E}$ \\
\hline$(\mathrm{o}+\mathrm{p})-\mathrm{H}_{2}{ }^{13} \mathrm{CO}$ & 200 & $1.1 \times 10^{16}$ & 0.34 & $\mathrm{E}$ \\
\hline$(\mathrm{o}+\mathrm{p})-\mathrm{D}_{2} \mathrm{CO}$ & 200 & $5.9 \times 10^{15}$ & 0.34 & $\mathrm{E}$ \\
\hline OCS & 200 & $2.0 \times 10^{18}$ & 0.32 & $\mathrm{E}$ \\
\hline $\operatorname{OCS} v_{2}=1$ & 200 & $2.4 \times 10^{16}$ & 0.32 & $\mathrm{E}$ \\
\hline $\mathrm{O}^{13} \mathrm{CS}$ & 200 & $3.1 \times 10^{16}$ & 0.32 & $\mathrm{E}$ \\
\hline $\mathrm{HC}_{3} \mathrm{~N}$ & 200 & $2.0 \times 10^{16}$ & 0.20 & $\mathrm{E}$ \\
\hline $\mathrm{HC}_{3} \mathrm{~N} v_{7}=1$ & 200 & $2.0 \times 10^{15}$ & 0.20 & $\mathrm{E}$ \\
\hline $\mathrm{DC}_{3} \mathrm{~N}^{*}$ & 200 & $3.1 \times 10^{14}$ & 0.20 & $\mathrm{G}$ \\
\hline SO & 200 & $\gg 2.4 \times 10^{15}$ & $\mathrm{BF}$ & $\mathrm{E}$ \\
\hline $\mathrm{S}^{18} \mathrm{O}$ & 200 & $<8.0 \times 10^{15}$ & 0.6 & $\mathrm{G}$ \\
\hline $\mathrm{SO}_{2}$ & 200 & $6.0 \times 10^{15}$ & $\mathrm{BF}$ & $\mathrm{G}$ \\
\hline${ }^{34} \mathrm{SO}_{2}$ & 200 & $2.7 \times 10^{14}$ & $\mathrm{BF}$ & $\mathrm{G}$ \\
\hline $\mathrm{HNCO}$ & 260 & $1.6 \times 10^{15}$ & $\mathrm{BF}$ & $\mathrm{E}$ \\
\hline $\mathrm{HN}^{13} \mathrm{CO}$ & 260 & $2.5 \times 10^{13}$ & $\mathrm{BF}$ & $\mathrm{E}$ \\
\hline$(\mathrm{o}+\mathrm{p})-\mathrm{H}_{2}{ }^{13} \mathrm{CS}$ & 200 & $1.2 \times 10^{15}$ & 0.60 & $\mathrm{E}$ \\
\hline$(\mathrm{o}+\mathrm{p})-\mathrm{HDCS}^{*}$ & 200 & $1.0 \times 10^{15}$ & 0.60 & E \\
\hline $\mathrm{HCOOH}$ & 100 & $9.5 \times 10^{18}$ & 0.12 & $\mathrm{E}$ \\
\hline $\mathrm{H}^{13} \mathrm{COOH}$ & 100 & $1.5 \times 10^{17}$ & 0.12 & $\mathrm{E}$ \\
\hline HCOOD $^{*}$ & 100 & $1.0 \times 10^{17}$ & 0.12 & $\mathrm{E}$ \\
\hline $\mathrm{CH}_{3} \mathrm{OCH}_{3}$ & 200 & $4.0 \times 10^{18}$ & 0.12 & $\mathrm{E}$ \\
\hline $\mathrm{CH}_{3} \mathrm{CHO} v_{t}=0$ & 100 & $4.0 \times 10^{15}$ & $\mathrm{BF}$ & $\mathrm{G}$ \\
\hline $\mathrm{CH}_{3} \mathrm{CHO} v_{t}=1$ & 100 & $5.6 \times 10^{14}$ & $\mathrm{BF}$ & G \\
\hline $\mathrm{CH}_{3} \mathrm{CHO} v_{t}=2$ & 100 & $1.1 \times 10^{14}$ & $\mathrm{BF}$ & $\mathrm{G}$ \\
\hline $\mathrm{aGg}^{\prime}-\left(\mathrm{CH}_{2} \mathrm{OH}\right)_{2}$ & 145 & $2.0 \times 10^{15}$ & $\mathrm{BF}$ & $\mathrm{G}$ \\
\hline$(\mathrm{o}+\mathrm{p})-\mathrm{H}_{2} \mathrm{CCO}^{*}$ & 200 & $6.7 \times 10^{14}$ & $\mathrm{BF}$ & $\mathrm{G}$ \\
\hline $\mathrm{CH}_{2} \mathrm{OHCHO}$ & 265 & $1.0 \times 10^{15}$ & $\mathrm{BF}$ & $\mathrm{G}$ \\
\hline $\mathrm{NH}_{2} \mathrm{CHO}^{*}$ & 200 & $3.0 \times 10^{14}$ & $\mathrm{BF}$ & $\mathrm{G}$ \\
\hline
\end{tabular}

Notes. ${ }^{(1)}$ Estimated column densities. ${ }^{(2)}$ Diameter of the emission region for the compact component assuming a disk morphology. ${ }^{(3)} \mathrm{BF}$ : Beam filling component. ${ }^{(4)} \mathrm{E}$ : The disk diameter estimated from our modeling. ${ }^{(5)} \mathrm{G}$ : only a guess because the size of the disk cannot be constrained on basis of our data. ${ }^{(6)}$ The fit to this component is not reliable because the lines suffers from spatial filtering and opacity effects (see text). ${ }^{(*)}$ Tentative detection.

also detected two lines of the OCS $v_{2}=1$ vibrational state at $E_{\mathrm{vib}}=748.8 \mathrm{~K}$ (see Table A.1). Assuming the same source size, the intensities of these lines imply a vibrational temperature, $T_{\text {vib }}=170 \mathrm{~K}$, in agreement with the rotational temperature we assumed. The same assumptions are used to fit the $\mathrm{OC}^{33} \mathrm{~S} J=19 \rightarrow 18$ line in FU05. 


\subsection{Sulfur monoxide: $S O, S^{18} \mathrm{O}$}

Only the intense line of SO at $219949 \mathrm{MHz}$ was detected. From its intensity and assuming $T_{\text {rot }}=200 \mathrm{~K}$, we derived a beam averaged SO column density of $2.4 \times 10^{15} \mathrm{~cm}^{-2}$. The emission of this line is extended (see Table 1 and Fig. 1) and, most likely, optically thick. There are no lines of ${ }^{34} \mathrm{SO},{ }^{33} \mathrm{SO}$, and/or $\mathrm{S}^{18} \mathrm{O}$ in the spectrum that could be used to determine the line opacity. As commented in Sect. 5 a possible detection of $\mathrm{S}^{18} \mathrm{O}$ in the spectrum published by FU05 suggests that the column density could be as much as a factor of $\sim 600$ higher (see Sect. 5 and Table 3). Unfortunately, this line is blended with other intense lines and this makes the estimate uncertain. We consider our fitted value as a lower limit to the real one.

\subsection{Sulfur dioxide: $\mathrm{SO}_{2},{ }^{34} \mathrm{SO}_{2}$}

One $\mathrm{SO}_{2}$ line at $219276 \mathrm{MHz}$ and two ${ }^{34} \mathrm{SO}_{2}$ lines at 219355 and $221115 \mathrm{MHz}$, respectively were detected. These lines are reasonably fitted using $T_{\text {rot }}=200 \mathrm{~K}$, a beam averaged $\mathrm{SO}_{2}$ column density of $6 \times 10^{15} \mathrm{~cm}^{-2}$ and ${ }^{32} \mathrm{~S} /{ }^{34} \mathrm{~S}=22$. The $\mathrm{SO}_{2}$ line is optically thin providing a good estimate of the total number of $\mathrm{SO}_{2}$ molecules within our beam. On the other hand, we could not estimate an accurate value of the opacity that would allow us to constrain the size of the emitting region; however, we checked that the $\mathrm{SO}_{2}$ line would be optically thin even if all the emission arises from the $0.1^{\prime \prime}$ compact core.

\subsection{Cyanoacetylene: $\mathrm{HCCCN}, \mathrm{HCCCN} v_{7}=1, \mathrm{DCCCN}$, HCCNC}

We detected one $\mathrm{HC}_{3} \mathrm{~N}$ line at $218325 \mathrm{MHz}$ and two lines of the vibrational state $v_{7}=1$ at $218861 \mathrm{MHz}$ and $219174 \mathrm{MHz}$, respectively, although the one at $219174 \mathrm{MHz}$ is blended with an intense ethanol line. In addition, we tentatively detected the $\mathrm{H}^{13} \mathrm{CCCN} 25 \rightarrow 24$ line. Unfortunately, the frequency of the $\mathrm{H}^{13} \mathrm{CCCN}$ line is close to that of the ${ }^{13} \mathrm{CO} 2 \rightarrow 1$ line and could have some contribution from its wing. Assuming that the isotopologue line is not blended with ${ }^{13} \mathrm{CO}$, we fit this line with $T_{\text {rot }}=200 \mathrm{~K}$ and $N\left(\mathrm{H}^{13} \mathrm{CCCN}\right)=8.8 \times 10^{14} \mathrm{~cm}^{-2}$ and a source size of $\sim 0.20^{\prime \prime}$ which implies an upper limit to the $\mathrm{HC}_{3} \mathrm{~N}$ column density of $<5.7 \times 10^{16} \mathrm{~cm}^{-2}$. This value, however, overestimates the intensity of the lines of the main isotopologue, which are better fitted with $N(\mathrm{HCCCN})=2.0 \times 10^{16} \mathrm{~cm}^{-2}$. We adopt this latter solution and conclude that our column density is accurate within a factor of 3 . There is a weak feature at $219490 \mathrm{MHz}$ that could correspond to DCCCN $J=$ $26 \rightarrow 25$. Since this line is very weak and there is no other DCCCN line in the spectrum that could confirm the detection, we will consider it as a tentative detection and use its intensity to derive an upper limit to the DCCCN column density of $N(\mathrm{DCCCN}) / N(\mathrm{HCCCN})<0.016$. We might have detected one line of HCCNC at $218558 \mathrm{MHz}$, but it is blended with an intense ethanol line. Therefore, we can only derive a lower limit to the $N(\mathrm{HCCCN}) / N(\mathrm{HCCNC})$ column density ratio of $>13$.

\subsection{Acetaldehyde: $\mathrm{CH}_{3} \mathrm{CHO}$}

Very few intense lines of $\mathrm{CH}_{3} \mathrm{CHO}$ are found in the observed wavelength range and some of them are blended with others from abundant species. We detected without blending the $220446 \mathrm{MHz}$ line of $\mathrm{E}-\mathrm{CH}_{3} \mathrm{CHO}$, and marginally the $221222 \mathrm{MHz}$ line of $\mathrm{A}-\mathrm{CH}_{3} \mathrm{CHO} v_{t}=1$, the $219780 \mathrm{MHz}$ line of $\mathrm{A}-\mathrm{CH}_{3} \mathrm{CHO} v_{t}=2$, and the $221216 \mathrm{MHz}$ line of E- $\mathrm{CH}_{3} \mathrm{CHO} v_{t}=2$. All these lines were fitted assuming $T_{\text {vib }}=100 \mathrm{~K}$. Making the additional assumption that $T_{\text {rot }}=T_{\text {vib }}$, we derived a total beam averaged $\mathrm{A}+\mathrm{E} \mathrm{CH}_{3} \mathrm{CHO}$ column density of $4 \times 10^{15} \mathrm{~cm}^{-2}$ in the $v=0$ ground state. In this case, the lines of the ${ }^{13} \mathrm{C}$ isotopologue were not detected. As for other oxygenated molecules, we assumed that the source is extended relative to our beam. Although the firm detections in the $218-221 \mathrm{GHz}$ frequency range are only a few, this model is fully confirmed by the good match to the data published by FU05 (see Sect. 5).

\subsection{Formic acid: $\mathrm{HCOOH}$}

Two lines of $\mathrm{HCOOH}$, one at $218938 \mathrm{MHz}$ and the other at $220038 \mathrm{MHz}$, were detected in our spectrum. However, their line intensities were not consistent with thermalized optically thin emission. Since the two lines have very similar $E_{u}$, this discrepancy cannot be due to a wrong rotation temperature. Spatial filtering cannot be the cause of this discrepancy, because such an intense line should have been detected in the $30 \mathrm{~m} \mathrm{spec}-$ trum. However, the Einstein spontaneous emission coefficient, $A_{i j}$, of the $220038 \mathrm{MHz}$ line is ten times lower than that of the $218938 \mathrm{MHz}$ line which results in a higher opacity of the former for the same physical conditions. We propose that the high opacity of the line at $220038 \mathrm{MHz}$ causes the anomalous line intensity ratio. The detection of $\mathrm{H}^{13} \mathrm{COOH}$ at $219341.849 \mathrm{MHz}$ also argues in favor of a high column density of $\mathrm{HCOOH}$ in this source. We can reasonably fit both lines of $\mathrm{HCOOH}$ and that of $\mathrm{H}^{13} \mathrm{COOH}$ assuming $T_{\text {rot }}=100 \mathrm{~K}$, a total $\mathrm{HCOOH}$ column density of $9.5 \times 10^{18} \mathrm{~cm}^{-2}$, and a source size of $\sim 0.1^{\prime \prime}$. The profile of the $\mathrm{H}^{13} \mathrm{COOH}$ line is wider than expected (see Fig. A.2), suggesting some contribution from the outflow or partial blending with other line. Because of this possible blending, the integrated intensity of this line is uncertain by a factor of 2 . We selected the solution that best fitted the $218938 \mathrm{MHz}$ line. The fit to the $\mathrm{HCOOH}$ line at $220038 \mathrm{MHz}$ is not perfect, but this is not worrying because our model is not adequate to reproduce the profile of very optically thick lines. The intensity of this line is not sensitive to the $\mathrm{HCOOH}$ column density in the range of column densities we are dealing with. Some $\mathrm{HCOOH}$ lines were also observed by FU05. As seen in Fig. 5, the lines are reasonably reproduced with our values. We have searched for the DCOOH and HCOOD lines in our spectrum and found one transition of HCOOD at $218541 \mathrm{MHz}$ from which we derive $N(\mathrm{HCOOD}) / N(\mathrm{HCOOH}) \approx 0.01$ as long as both species come from the same region. Since we have only one line of HCOOD and it is at $4 \sigma$, we indicated this detection as tentative in Table 3.

\subsection{Dimethyl ether: $\mathrm{CH}_{3} \mathrm{OCH}_{3}$}

We detected four unblended lines of $\mathrm{CH}_{3} \mathrm{OCH}_{3}$, at 218492, 219301, 220847, and $220893 \mathrm{MHz}$. The other lines of this species are blended with intense lines. Assuming that the source is filling the beam, we derived $T_{\text {rot }}=500 \mathrm{~K}$ and $N\left(\mathrm{CH}_{3} \mathrm{OCH}_{3}\right)=2 \times 10^{16} \mathrm{~cm}^{-2}$. We considered that this apparently high rotation temperature was an opacity effect and obtained a better fit assuming $T_{\text {rot }}=200 \mathrm{~K}$, $N\left(\mathrm{CH}_{3} \mathrm{OCH}_{3}\right)=4 \times 10^{18} \mathrm{~cm}^{-2}$ and a source diameter of $0.1^{\prime \prime}$. These parameters are consistent with the non-detection of the ${ }^{13} \mathrm{C}$ isotopologues and we adopted them. 


\subsection{Ethylene glycol: aGg'-( $\left(\mathrm{CH}_{2} \mathrm{OH}\right)_{2}, g G g^{\prime}-\left(\mathrm{CH}_{2} \mathrm{OH}\right)_{2}$}

The aGg' conformer of ethylene glycol, together with methyl formate, is one of those with the highest number of lines in our spectrum. We detected twelve lines, and seven were unblended. We used these seven unblended lines to derive a rotation temperature of $145 \mathrm{~K}$ and a beam averaged column density of $N(\mathrm{aGg}$ $\left.\left(\mathrm{CH}_{2} \mathrm{OH}\right)_{2}\right)=2.3 \times 10^{15} \mathrm{~cm}^{-2}$ (see Fig. 2 and Table 2). The line at $218576 \mathrm{MHz}$ was removed from the final fit since it was very far from the intensity traced by the other lines (see Fig. 2) and is certainly blended with something else. In this case, we did not have information about the transitions of the ${ }^{13} \mathrm{C}$ isotopologues and deuterated species. The emission is compatible with the beam filling assumption, although we cannot discard a smaller size.

We also searched for $\mathrm{gGg}^{\prime}-\left(\mathrm{CH}_{2} \mathrm{OH}\right)_{2}$ in our spectrum. We have a good match with eight lines that can be assigned to this species $(218712,219097,219389,219410,220763,220448$, $220887,221596 \mathrm{MHz}$ ). Modeling the spectrum, we obtain that five additional lines are also present in our data but blended with other species. However there are two lines, at $218144 \mathrm{MHz}$ and $220249 \mathrm{MHz}$, that should have been detected and do not appear in the spectrum. These lines have similar energies to the previous ones and their absence cannot be explained with a simple excitation argument. This species has not been detected in any other source thus far. Waiting for confirmation, we do not include it in the model.

\subsection{Ketene: $\mathrm{H}_{2} \mathrm{CCO}$}

We detected one o- $\mathrm{H}_{2} \mathrm{CCO}$ line at $220177 \mathrm{MHz}$ and one p- $\mathrm{H}_{2} \mathrm{CCO}$ at $221545 \mathrm{MHz}$ with similar intensity. The first transition is at $E_{u}=62.4 \mathrm{~K}$ and the second one $E_{u}=894.8 \mathrm{~K}$. It is not possible to fit both lines assuming reasonable physical conditions and optically thin emission. One possibility is that the line at $221545 \mathrm{MHz}$ is not ketene. In this case, we can fit the other line assuming a rotation temperature of $T_{\text {rot }}=200 \mathrm{~K}$ and a beam averaged ortho+para $\mathrm{H}_{2} \mathrm{CCO}$ column density of $6.7 \times 10^{14} \mathrm{~cm}^{-2}$, where we have assumed an ortho-to-para ratio of 3 . Another possibility is that the emission is optically thick in both lines. In this case, we need a ketene column density of $4.0 \times 10^{18} \mathrm{~cm}^{-2}$ in a compact $\sim 0.1^{\prime \prime}$ region. There is no line of the ${ }^{13} \mathrm{C}$ isotopologue in the observed frequency range that would allow us to determine the opacities. The deuterated compounds have no intense transitions in the observed wavelength range either. We selected the optically thin case adopting a conservative approach. Taking into account the uncertainty in its column density, we do not use this species in our comparison with other sources.

\subsection{Glycolaldehyde: $\mathrm{CH}_{2} \mathrm{OHCHO}$, Formamide: $\mathrm{NH}_{2} \mathrm{CHO}$}

There are thirteen intense lines of glycolaldehyde in the sampled wavelength range. From these, six are not blended with other features and do not have any other likely identification. Assuming $T_{\text {rot }}=200 \mathrm{~K}$, we estimated a beam averaged column density of $1.0 \times 10^{15} \mathrm{~cm}^{-2}$. Only one line of $\mathrm{NH}_{2} \mathrm{CHO}$ lies in our spectrum. It is at $218459 \mathrm{MHz}$ and is blended with a line of T-ethanol. Our fit to T-ethanol failed to predict the high intensity of the observed feature suggesting some contribution from $\mathrm{NH}_{2} \mathrm{CHO}$. The total intensity would be consistent with a beam averaged formamide column density of $3.0 \times 10^{14} \mathrm{~cm}^{-2}$.

\subsection{Vinyl cyanide: $\mathrm{CH}_{2} \mathrm{CHCN}$}

We detected two rotational lines, at $218675 \mathrm{MHz}$ and $220345 \mathrm{MHz}$, that could correspond to $\mathrm{CH}_{2} \mathrm{CHCN}$. The intense line at $220345 \mathrm{MHz}$ is overlapped with one of $\mathrm{CH}_{3} \mathrm{CN}$. Some features of the $v_{11}=1$ vibrational state at an energy of $328.4 \mathrm{~K}$ could have also been detected but are blended with other more intense lines. Since we could not determine the rotation temperature, we assumed the fixed value of $T_{\text {rot }}=200 \mathrm{~K}$ and the same source size as the chemically related species $\mathrm{CH}_{3} \mathrm{CN}$, i.e., a diameter of $\sim 0.1^{\prime \prime}$, but our fit was very bad. The relative intensities of the lines at $220345 \mathrm{MHz}$ and $218675 \mathrm{MHz}$ cannot be accounted for by LTE models. We were not able to obtain a better fit by varying the rotation temperature and/or the source size. One possibility is that the intensity of one of the lines is incorrect because of baseline uncertainties or spatial filtering. The second possibility is that our identification is false. Since we do not have a better candidate for the $220345 \mathrm{MHz}$ line, we derived an upper limit to the vinyl cyanide column density using its line intensity and fixing the rotation temperature to $200 \mathrm{~K}$. This compound is quite abundant in massive hot cores (see López et al. 2014), and this upper limit is interesting for comparison with these objects.

\section{The hot core model}

Based on the calculations explained in Sect. 4, we propose the hot core model shown in Table 3. This model is not unique and some parameters, especially source sizes, are quite uncertain. However, it cannot be done better. Higher spatial resolution is required to better constrained the spatial structure. Fortunately, the total number of molecules in our beam is well constrained, especially when we have detected the ${ }^{13} \mathrm{C}$ isotopologue or the emision is optically thin, which is the case for most species. Our model is self-consistent; i.e., once a molecule is included, its isotopologues and deuterated compounds are also included. For isotopologues, we assume ${ }^{12} \mathrm{C} /{ }^{13} \mathrm{C}=65$, ${ }^{16} \mathrm{O} /{ }^{18} \mathrm{O}=650,{ }^{32} \mathrm{~S} /{ }^{34} \mathrm{~S}=22$, and ${ }^{34} \mathrm{~S} /{ }^{33} \mathrm{~S}=6$ (Chin et al. 1995; Milam et al. 2005). The column densities of the deuterated species are calculated independently to determine the deuterium fractions. When possible we directly compare the deuterated compound with the ${ }^{13} \mathrm{C}$ isotopologue, which is expected to have similar fractional abundance, in order to avoid opacity effects in the derived column density ratio. The obtained values of deuterium fractions are $\sim 0.06$ for methyl formate, $\sim 0.02$ for methanol, $\sim 0.008$ for the doubly deuterated formaldehyde, $\sim 0.01$ for $\mathrm{DC}_{3} \mathrm{~N}$, and $<0.01$ for HCOOD (see Table 3). These are average values in the hot core since the hydrogenated and deuterated species could have different spatial distributions. Using MADEX, we have synthesized the total spectrum assuming LTE, the parameters shown in Table 3 and convolving with a circular beam of $\sim 1.4^{\prime \prime}$. The obtained spectrum is compared with observations in Fig. A.1. Our fit is reasonably good taking into account the simplicity of our model. There are, however, two frequency ranges, 218.0-219.12 GHz and 221.46-221.62, where the agreement is poor. One reason is possible observational errors. We note that the second region is at the end of the observed frequency range where the passband calibration is more uncertain, but the existence of intense $U$ lines suggests the existence of a population of COMs that have not been identified yet.

An important test for our model is to reproduce the interferometric observations published by FU05 with a higher angular resolution of $0.63^{\prime \prime} \times 0.46^{\prime \prime}$. We synthesized the spectrum assuming the same parameters as in Table 3 but convolving with 
a smaller beam, $\sim 0.52^{\prime \prime}$, and compared with FU05 observations (see Fig. A.5). We find a remarkably good agreement for most of the species which confirmed that our model was not far from reality.

We identified the line at $231143 \mathrm{MHz}$ in FU05 as $\mathrm{H}_{2}^{13} \mathrm{CS}$ providing an estimate of the $\mathrm{H}_{2} \mathrm{CS}$ abundance in the hot core (see Table 3). However, we have only one line of the ${ }^{13} \mathrm{C}$ isotopologue and the identification could be wrong. A similar situation occurs for $\mathrm{S}^{18} \mathrm{O}$. We have detected one line at $228272 \mathrm{MHz}$, but it is blended with intense $\mathrm{CH}_{3} \mathrm{OCHO}$ and $\mathrm{CH}_{3} \mathrm{OCOD}$ features. From this line we estimate a $\mathrm{S}^{18} \mathrm{O}$ column density of $8 \times 10^{15} \mathrm{~cm}^{-2}$ within the $\sim 0.52^{\prime \prime}$ ( $\left.\sim 650 \mathrm{AU}\right)$ beam. Because of the blending, this estimate is model-dependent and suffers from larger uncertainty.

An important discrepancy between our model and FU05 observations comes from $\mathrm{G}+$ ethanol. There are two predicted G+ethanol lines, $228491 \mathrm{MHz}$ and $228560 \mathrm{MHz}$ that do not appear in the FU05 spectrum while all the T-ethanol lines are correctly reproduced. One possibility is that we have a lower kinetic temperature than assumed since the $\mathrm{G}+$ ethanol is $60 \mathrm{~K}$ higher in energy than T-ethanol. But the temperature would have to be unreasonably low, $<50 \mathrm{~K}$, to reproduce the observations. These lines are close to the edge of the spectral band and could suffer from some instrumental effects.

In Table A.2 we give a list with our new identifications of the data published by FU05. In this work we have identified more lines and found some misidentifications. The lines previously identified as $\mathrm{c}_{-} \mathrm{C}_{3} \mathrm{D}$ are instead carried by $\mathrm{CH}_{3} \mathrm{CHO}$. In fact, $\mathrm{CH}_{3} \mathrm{CHO}$ is the carrier of most of the lines in the band centered at $231.3 \mathrm{GHz}$. We have a handful of lines around the ${ }^{13} \mathrm{CS}$ frequency without any plausible identification. One possibility is that we are observing high velocity gas emitting in the ${ }^{13} \mathrm{CS}$ $5 \rightarrow 4$ line, but this would imply an outflow with velocities as high as $50 \mathrm{~km} \mathrm{~s}^{-1}$. We have not seen such high velocities in the CO single-dish and interferometric spectra (Fuente et al. 2001, FU05). Therefore, we think that there are still some unidentified lines at these frequencies. The intense lines at $228307 \mathrm{MHz}$, $228363 \mathrm{MHz}, 228427 \mathrm{MHz}$, and $228467 \mathrm{MHz}$, although identified, are poorly reproduced by our model. We have not found any simple way to improve the agreement between our model and observations. Either these lines are far from the LTE approximation or we are still missing important species. Additional observations could help to discern their origin.

The observations presented in this paper have significantly contributed to the understanding of the spectra published by FU05 proving that observations in a large range of frequencies are needed for the correct line identification.

\section{Discussion}

\subsection{Physical conditions}

It is known that hot cores are heterogenous objects where different species come from different regions with different physical conditions (see, e.g., Beuther et al. 2011; Brouillet et al. 2013; Bell et al. 2014). For this reason, we only compare rotation temperatures measured with the same molecular species.

Methyl cyanide is easily thermalized for the densities prevailing in hot cores and hence a good thermometer of the molecular gas. The rotational diagram shows that the temperature in the FIRS 2 hot core is $\approx 400 \mathrm{~K}$. This temperature is similar to that measured in massive hot cores. Beuther et al. (2011) proposed the existence of a warm component of $600 \pm 200 \mathrm{~K}$ in the Orion hot core based on submillimeter $\mathrm{CH}_{3} \mathrm{CN}$ lines as observed with the Submillimeter Array (SMA). More recently, Bell et al. (2014) estimated a rotation temperature of $\sim 220 \mathrm{~K}$ towards IRc2 and $\sim 400 \mathrm{~K}$ in the hot spot located $\sim 14^{\prime \prime} \mathrm{NE}$ using data from the $30 \mathrm{~m}$ telescope. Lower temperatures, however, are found in hot corinos. Bisschop et al. (2008) derived an upper limit of $<390 \mathrm{~K}$ in IRAS 16293-2422 A and B based on interferometric SMA data. Fuente et al. (2009) derived a rotation temperature of $97 \pm 25 \mathrm{~K}$ in the hot core located in the IM protocluster IC $1396 \mathrm{~N}$. A similar rotation temperature, $\sim 100 \mathrm{~K}$, was derived by Sánchez-Monge et al. (2010) in the hot core associated with IRAS 22198+6336. Although the luminosity of FIRS 2 is similar, our observations suggest the existence of a warmer gas component ( 400 K) in FIRS 2 than in IC $1396 \mathrm{~N}$ and IRAS $22198+6336$. IC $1396 \mathrm{~N}$ is a protocluster with three young stellar objects (YSOs). Although the total luminosity is similar to that of FIRS 2, the luminosity of each YSO is lower and probably closer to that of an Ae star, i.e., $<100 L_{\odot}$. IRAS $22198+6336$ is morphologically similar to FIRS 2. Sánchez-Monge et al. (2010) detected only the K lines with $E_{u}=100-300 \mathrm{~K}$, while we used lines with $E_{u}>600 \mathrm{~K}$ in this study. It is possible that a warmer component remains hindered in IRAS $22198+6336$ and would require higher sensitivity observations of transitions with higher $E_{u}$ to be detected. In fact, FU05 determined a gas temperature of $50-100 \mathrm{~K}$ in FIRS 2 from lower sensitivity observations of the $\mathrm{CH}_{3} \mathrm{CN} 5_{K} \rightarrow 4_{K}$ ladder, from lines with $E_{u}=13-154 \mathrm{~K}$.

The rotational diagrams of the oxygenated species give lower rotation temperatures than with $\mathrm{CH}_{3} \mathrm{CN}$. We derived rotation temperatures between $100 \mathrm{~K}$ and $200 \mathrm{~K}$ from the $\mathrm{CH}_{2} \mathrm{DOH}$, $\mathrm{CH}_{3} \mathrm{CHO}$, and $\mathrm{aGg}{ }^{\prime}-\left(\mathrm{CH}_{3} \mathrm{OH}\right)_{2}$ lines and between $200 \mathrm{~K}$ and $300 \mathrm{~K}$ from $\mathrm{CH}_{3} \mathrm{OCHO}$ and $\mathrm{HNCO}$. Palau et al. (2011) derived rotation temperatures of $\sim 100-150 \mathrm{~K}$ in the IM hot cores in IRAS 22198+6336 and AFGL 5142 from the ethanol lines. Rotation temperatures of around $100 \mathrm{~K}$ are also derived in Orion from $\mathrm{CH}_{3} \mathrm{OCH}_{3}$, methyl formate, deuterated methanol, and OCS (Tercero et al. 2010; Peng et al. 2012; Brouillet et al. 2013). Therefore, we do not detect significant differences between the rotation temperatures measured with these O-bearing species between IM and massive hot cores. Lower rotation temperatures, $\sim 50 \mathrm{~K}$, are measured with $\mathrm{CH}_{3} \mathrm{OCHO}$ in IRAS 16293-2422 (Pineda et al. 2012).

In Table 4, we show a summary of the rotation temperatures derived from different molecules and for different hot cores. The comparison is not totally fair since $\mathrm{CH}_{3} \mathrm{CN}$ and the O-bearing molecules could come from different regions. This is the case of IRAS 16293-2422, where $\mathrm{CH}_{3} \mathrm{CN}$ comes mainly from the southern component $(\mathrm{A})$ and the $\mathrm{O}$-bearing molecules from the northern one (B) (Bisschop et al. 2008). As commented above, different regions are associated with $\mathrm{O}$ - and $\mathrm{N}$-bearing molecules in Orion. Towards the other hot cores, the spatial resolution provided by the existing observations is lower, preventing us from resolving regions with different chemistries. Nevertheless, it seems that there is a weak trend with the maximum gas temperature increasing from low to massive hot cores as measured with $\mathrm{CH}_{3} \mathrm{CN}$. There is no trend for the O-bearing molecules.

This suggests a scenario in which the O-bearing molecules are more abundant in extended regions with temperatures $\sim 100 \mathrm{~K}$ in both hot cores and corinos. Instead, $\mathrm{CH}_{3} \mathrm{CN}$ probes the hottest region of the core, and its rotation temperature increases with the luminosity. Of course, there is a problem with the spatial scales that are different for low-mass and massive stars, but, taking into account that massive stars are more distant, this would favor the interpretation of higher temperatures in the massive stars. Therefore, within the limitations of 
Table 4. Comparison of rotation temperatures between hot cores and hot corinos.

\begin{tabular}{|c|c|c|c|c|}
\hline Hot core/corino & $L_{\odot}$ & $T_{\text {rot }}\left(\mathrm{CH}_{3} \mathrm{CN}\right)$ & $T_{\text {rot }}(\mathrm{X})$ & References \\
\hline IRAS 16293-2422 & $6.9-23$ & $\sim 150-<390 \mathrm{~K}$ & $\sim 60 \mathrm{~K}\left(\mathrm{CH}_{3} \mathrm{CHO}\right)$ & $\begin{array}{l}\text { Cazaux et al. (2003), Bisschop et al. (2008) } \\
\text { Pineda et al. (2012) }\end{array}$ \\
\hline IC $1396 \mathrm{~N}$ & $<300$ & $\sim 100 \mathrm{~K}$ & & Neri et al. (2007), Fuente et al. (2009) \\
\hline IRAS $22198+6336$ & 370 & $\sim 100 \mathrm{~K}$ & $\sim 120 \mathrm{~K}\left(\mathrm{CH}_{3} \mathrm{CH}_{2} \mathrm{OH}\right)$ & Sánchez-Monge et al. (2010), Palau et al. (2011) \\
\hline NGC 7129 FIRS 2 & 480 & $\sim 400 \mathrm{~K}$ & $\sim 120 \mathrm{~K}\left(\mathrm{aGg}-\left(\mathrm{CH}_{2} \mathrm{OH}\right)_{2}\right)$ & This work \\
\hline AFGL 5142 & 2300 & & $\sim 140-210 \mathrm{~K}\left(\mathrm{CH}_{3} \mathrm{CH}_{2} \mathrm{OH}\right)$ & Palau et al. (2011) \\
\hline Orion KL & $\sim 10^{4}$ & $400-600 \mathrm{~K}$ & $\sim 100-200 \mathrm{~K}\left(\mathrm{CH}_{3} \mathrm{OCH}_{3}, \mathrm{OCS}\right)$ & $\begin{array}{l}\text { Tercero et al. (2010), Beuther et al. (2011), } \\
\text { Brouillet et al. (2013), Bell et al. (2014) }\end{array}$ \\
\hline
\end{tabular}

Table 5. Average fractional abundances.

\begin{tabular}{lcc}
\hline \hline & $T_{\text {dust }}=50 \mathrm{~K}$ & $T_{\text {dust }}=400 \mathrm{~K}$ \\
\hline$M_{\text {gas }+ \text { dust }}\left(M_{\odot}\right)$ & 1.8 & 0.46 \\
$N_{\mathrm{H}_{2}}\left(\mathrm{~cm}^{-2}\right)$ & $2.0 \times 10^{24}$ & $5.0 \times 10^{23}$ \\
\hline $\mathrm{CH}_{3} \mathrm{CN}\left({ }^{13} \mathrm{C}\right)^{1}$ & $6 \times 10^{-9}$ & $3 \times 10^{-8}$ \\
$\mathrm{CH}_{3} \mathrm{OCHO}$ & $2 \times 10^{-8}$ & $7 \times 10^{-8}$ \\
$\mathrm{CH}_{3} \mathrm{CH}_{2} \mathrm{CN}$ & $4 \times 10^{-10}$ & $2 \times 10^{-9}$ \\
$\mathrm{CH}_{2} \mathrm{CHCN}$ & $<3 \times 10^{-9}$ & $<2 \times 10^{-8}$ \\
$\mathrm{CH}_{3} \mathrm{OH}\left({ }^{13} \mathrm{C}\right)$ & $1 \times 10^{-6}$ & $5 \times 10^{-6}$ \\
$\mathrm{CH}_{2} \mathrm{DOH}$ & $7 \times 10^{-9}$ & $3 \times 10^{-8}$ \\
$\mathrm{CH}_{3} \mathrm{CH}{ }_{2} \mathrm{OH}$ & $1 \times 10^{-8}$ & $4 \times 10^{-8}$ \\
$\mathrm{CH}_{3} \mathrm{COCH}$ & $5 \times 10^{-10}$ & $2 \times 10^{-9}$ \\
$\mathrm{H}_{2} \mathrm{CO}\left({ }^{13} \mathrm{C}\right)$ & $2 \times 10^{-8}$ & $8 \times 10^{-8}$ \\
$\mathrm{D}_{2} \mathrm{CO}$ & $2 \times 10^{-10}$ & $7 \times 10^{-10}$ \\
$\left.\mathrm{OCS}^{13}{ }^{13} \mathrm{C}\right)$ & $5 \times 10^{-8}$ & $2 \times 10^{-7}$ \\
$\mathrm{HC}_{3} \mathrm{~N}$ & $2 \times 10^{-10}$ & $8 \times 10^{-10}$ \\
$\mathrm{SO}_{2}$ & $3 \times 10^{-9}$ & $1 \times 10^{-8}$ \\
$\mathrm{HNCO} \mathrm{H}_{2} \mathrm{CS}\left({ }^{13} \mathrm{C}\right)$ & $8 \times 10^{-10}$ & $3 \times 10^{-9}$ \\
$\left.\mathrm{HCOOH}^{13} \mathrm{C}\right)$ & $7 \times 10^{-9}$ & $3 \times 10^{-8}$ \\
$\mathrm{CH}_{3} \mathrm{OCH}$ & $3 \times 10^{-8}$ & $1 \times 10^{-7}$ \\
$\mathrm{CH}_{3} \mathrm{CHO}$ & $1 \times 10^{-8}$ & $6 \times 10^{-8}$ \\
$\mathrm{aGg}^{\prime}-\left(\mathrm{CH}{ }_{2} \mathrm{OH}\right)_{2}$ & $2 \times 10^{-9}$ & $1 \times 10^{-8}$ \\
$\mathrm{CH}_{2} \mathrm{OHCHO}$ & $5 \times 10^{-9}$ & $4 \times 10^{-9}$ \\
\hline
\end{tabular}

Notes. Average fractional abundance in a disk with a diameter of $\sim 1.4^{\prime \prime}$. ${ }^{(1)}$ Derived from the ${ }^{13} \mathrm{C}$ isotopologue assuming ${ }^{12} \mathrm{C} /{ }^{13} \mathrm{C}=65$.

our analysis, we can conclude that there is a trend of increasing temperature from hot corinos to massive hot cores. This trend is not so clear between IM and massive stars. As we have already discussed, there is no obvious difference between FIRS 2 and Orion.

Another important parameter is the total amount of gas in the hot core. One possibility is to calculate it from the dust continuum emission assuming a dust temperature and a dust emissivity value. FU05 adopted a dust temperature of $T_{\text {dust }}=100 \mathrm{~K}$ and $\kappa_{v}=0.015(1300 / \lambda(\mu \mathrm{m})) \mathrm{cm}^{2} \mathrm{~g}^{-1}$ and derived a total gas+dust mass of $2 M_{\odot}$ in the hot core FIRS 2. More recently, Palau et al. (2013) derived a similar value $\left(1.8 M_{\odot}\right)$ assuming $T_{\text {dust }}=50 \mathrm{~K}$ and $\kappa_{1.3 \mathrm{~mm}}=0.00899 \mathrm{~cm}^{2} \mathrm{~g}^{-1}$. Our results suggest that at least a fraction of the dust must be at $T_{\text {dust }}=400 \mathrm{~K}$. Assuming $T_{\text {dust }}=400 \mathrm{~K}$ and $\kappa_{1.3 \mathrm{~mm}}=0.01 \mathrm{~cm}^{2} \mathrm{~g}^{-1}$, we obtain a lower limit to the gas+dust mass of $0.46 M_{\odot}$. Therefore, the total gas+dust mass of the FIRS 2 hot core is uncertain in a factor of $\sim 4$. In Table 5 we show the average molecular abundances in a region of $1.4^{\prime \prime}$ assuming $M=1.8 M_{\odot}$ and $0.46 M_{\odot}$ for the hot core mass, respectively. When detected, we used the ${ }^{13} \mathrm{C}$ isotopologue to determine the total average molecular column density which is more reliable. As discussed in Sect. 4, the uncertainties in the molecular column densities would add an error of a factor $\leq 4-5$ to these values.

\subsection{Comparison with the Orion hot core}

The hot core associated with FIRS 2 is extraordinarily rich in complex molecules. We have detected glycolaldehyde and tentatively formamide; these compounds had only been detected in a few objects thus far. Glycolaldehyde was detected in Sgr B2 (Hollis et al. 2000; Halfen et al. 2006; Requena-Torres et al. 2008), the massive hot core G31.41+0.31 (Beltrán et al. 2009) and more recently towards the hot corino IRAS 16293-2422B (Jorgensen et al. 2012; Zapata et al. 2013). Formamide has been detected in Orion (Motiyenko et al. 2012), Sgr B2 (Belloche et al. 2013), a handful of massive prototypical hot cores (Adande et al. 2013), and IRAS 16293-2422 (Kahane et al. 2013). In Fig. A.1, we compare the FIRS 2 spectrum with that of the Orion hot core as observed with the $30 \mathrm{~m}$ telescope (Tercero et al. 2010). Surprisingly, there is a good match between the spectra, in number of lines and relative line intensities, suggesting that both hot cores have similar chemical characteristics. Of course, this is a qualitative comparison and we need to put it on quantitative grounds to establish firm conclusions.

In Table 6 and Fig. 3 we compare the molecular column densities in FIRS 2 and Orion KL. For this comparison, in FIRS 2, we have used the average column densities in a region of 1.4". The reason is that the sizes of the emitting regions are not determined for most of the species (see Table 3). One needs to know the rotation temperature and the line opacities independently, to be able to determine the source size. In many cases we have not detected the ${ }^{13} \mathrm{C}$ isotopologue that would allow us to derive the line opacities. In others, the number of unblended lines is not sufficient to determine the rotation temperature and we had to assume a reasonable value. On the contrary, the number of molecules per beam is a quite well determined parameter as long as the emission is optically thin which is a reasonable assumption for many complex species. When detected, we used the ${ }^{13} \mathrm{C}$ isotopologue to determine the total average column density.

Towards Orion KL, the molecular emission is formed of four physically different components: hot core, plateau, compact ridge, and extended ridge. The plateau has a chemistry characteristic of a shocked region and is considered to be associated with the shocks produced by a past eruptive episode. The extended ridge is the parent cloud that hosts the cluster of young stars. The compact ridge is a small $\left(\sim 15^{\prime \prime}\right) U$-shaped feature that is characterized as being very rich in complex O-bearing molecules. The hot core is a very compact source $\left(6^{\prime \prime}-10^{\prime \prime}\right)$ particularly rich in complex N-bearing molecules. We cannot resolve different regions within the FIRS 2 hot core; therefore, we consider that the FIRS 2 hot core is comparable to the hot core (HC) and the compact ridge $(\mathrm{CR})$ Orion components together. In Table 6 we show 
Table 6. Comparison between FIRS 2 and Orion KL.

\begin{tabular}{|c|c|c|c|}
\hline & $\begin{array}{l}\text { FIRS } 2^{1} \\
N\left(\mathrm{~cm}^{-2}\right)\end{array}$ & $\begin{array}{c}\text { Orion } \mathrm{KL}^{2} \\
N\left(\mathrm{~cm}^{-2}\right)\end{array}$ & References $^{3}$ \\
\hline $\mathrm{CH}_{3} \mathrm{CN}$ & $1.3 \times 10^{16}\left({ }^{13} \mathrm{C}\right)^{4}$ & $1.2 \times 10^{16}\left({ }^{13} \mathrm{C}\right)$ & Bell et al. (2014) \\
\hline $\mathrm{CH}_{3} \mathrm{OCHO}$ & $3.7 \times 10^{16}$ & $2.9 \times 10^{17}\left({ }^{13} \mathrm{C}\right)$ & Carvajal et al. (2009), Haykal et al. (2014) \\
\hline $\mathrm{CH}_{3} \mathrm{OCDO}$ & $1.1 \times 10^{15}$ & $1.0 \times 10^{15}$ & Margulès et al. (2010) \\
\hline $\mathrm{CH}_{3} \mathrm{CH}_{2} \mathrm{CN}$ & $8.8 \times 10^{14}$ & $2.3 \times 10^{16}\left({ }^{13} \mathrm{C}\right)$ & Daly et al. (2013) \\
\hline $\mathrm{CH}_{2} \mathrm{CHCN}$ & $<6.9 \times 10^{15}$ & $2.4 \times 10^{15}$ & López et al. (2014) \\
\hline $\mathrm{CH}_{3} \mathrm{OH}$ & $2.5 \times 10^{18}\left({ }^{13} \mathrm{C}\right)$ & $1.1 \times 18^{18}\left({ }^{13} \mathrm{C}\right)$ & Kolesniková et al. (2014) \\
\hline $\mathrm{CH}_{2} \mathrm{DOH}$ & $1.4 \times 10^{16}$ & $1.5 \times 10^{15}$ & Neill et al. (2013) \\
\hline $\mathrm{CH}_{3} \mathrm{CH}_{2} \mathrm{OH}$ & $2.2 \times 10^{16}$ & $3.6 \times 10^{16}$ & Kolesniková et al. (2014) \\
\hline $\mathrm{H}_{2} \mathrm{CO}$ & $4.1 \times 10^{16}\left({ }^{13} \mathrm{C}\right)$ & $4.3 \times 10^{16}\left({ }^{13} \mathrm{C}\right)$ & Neill et al. (2013) \\
\hline $\mathrm{D}_{2} \mathrm{CO}$ & $3.4 \times 10^{14}$ & $4.2 \times 10^{13}$ & Turner, B.E. (1990) \\
\hline OCS & $1.0 \times 10^{17}\left({ }^{13} \mathrm{C}\right)$ & $4.2 \times 10^{16}\left({ }^{13} \mathrm{C}\right)$ & Tercero et al. (2010) \\
\hline $\mathrm{HC}_{3} \mathrm{~N}$ & $4.1 \times 10^{14}$ & $7.7 \times 10^{15}\left({ }^{13} \mathrm{C}\right)$ & Esplugues et al. (2013b) \\
\hline $\mathrm{S}^{18} \mathrm{O}$ & $<1.5 \times 10^{15}$ & $2.2 \times 10^{14}$ & Esplugues et al. (2013a) \\
\hline $\mathrm{SO}_{2}$ & $6.0 \times 10^{15}$ & $1.1 \times 10^{17}$ & Esplugues et al. (2013a) \\
\hline $\mathrm{HNCO}$ & $1.6 \times 10^{15}$ & $5.5 \times 10^{15}\left({ }^{13} \mathrm{C}\right)$ & Marcelino et al. (2009) \\
\hline $\mathrm{H}_{2} \mathrm{CS}$ & $1.4 \times 10^{16}\left({ }^{13} \mathrm{C}\right)$ & $3.3 \times 10^{15}\left({ }^{13} \mathrm{C}\right)$ & Tercero et al. (2010) \\
\hline HDCS & $1.8 \times 10^{14}$ & $9.5 \times 10^{13}$ & Tercero et al. (2010) \\
\hline $\mathrm{HCOOH}$ & $6.9 \times 10^{16}\left({ }^{13} \mathrm{C}\right)$ & $4.9 \times 10^{13}$ & Turner, B.E. (1991) \\
\hline $\mathrm{CH}_{3} \mathrm{OCH}_{3}$ & $2.9 \times 10^{16}$ & $9.0 \times 10^{15}$ & Comito et al. (2005) \\
\hline $\mathrm{CH}_{3} \mathrm{CHO}$ & $5.0 \times 10^{15}$ & $2.8 \times 10^{14}$ & Turner (1991) \\
\hline $\mathrm{NH}_{2} \mathrm{CHO}^{*}$ & $3.0 \times 10^{14}$ & $9.6 \times 10^{14}$ & Motiyenko et al. (2012) \\
\hline
\end{tabular}

Notes. ${ }^{(1)}$ Average molecular column densities in a disk with a diameter of $1.4^{\prime \prime}$. It is calculated with the expression $N=N_{\mathrm{c}} \times\left(D\left({ }^{\prime \prime}\right) / 1.4^{\prime \prime}\right)^{2}+N_{\mathrm{ex}}$, where $N_{\mathrm{c}}$ and $D$ are the column density and size (diameter) of the compact component, respectively, and $N_{\text {ex }}$ is the column density of the beam filling component as shown in Table 3. ${ }^{(2)}$ Average molecular column densities considering a region of $15^{\prime \prime}$ of diameter towards Orion KL. We assume a beam filling factor of 0.44 for the hot core component $\left(\right.$ size $\left.=10^{\prime \prime}\right)$. ${ }^{(3)}$ References for the Orion column densities. ${ }^{(4)}$ Derived from the ${ }^{13} \mathrm{C}$ isotopologues assuming ${ }^{12} \mathrm{C} /{ }^{13} \mathrm{C}=65$ for FIRS 2 and ${ }^{12} \mathrm{C} /{ }^{13} \mathrm{C}=50$ for Orion.

the average column densities of the $\mathrm{HC}+\mathrm{CR}$ Orion components in a region of $15^{\prime \prime}$. To estimate this value, we have summed up the column densities of the HC and CR weighted by their respective filling factors: 0.44 for $\mathrm{HC}$ (assuming a size of 10") and 1 for the CR. When possible, we used the ${ }^{13} \mathrm{C}$ isotopologues to determine the Orion column densities (see Table 6). We assume ${ }^{12} \mathrm{C} /{ }^{13} \mathrm{C}=50$ for Orion (Comito et al. 2005; Tercero et al. 2010). Some authors, like Neill et al. (2013) and Comito et al. (2005) used a size of $10^{\prime \prime}$ for the CR. We have multiplied their column densities by 0.44 to account for the different adopted source sizes. For $\mathrm{CH}_{3} \mathrm{CHO}$ we have adopted the value of Turner (1991). This author did not distinguish among the different Orion components (hot core, plateau, compact ridge, and extended ridge) and we have taken the total value. For this reason this point is drawn as an empty circle in Fig. 3. In the case of $\mathrm{D}_{2} \mathrm{CO}$, Turner (1990) assumed a source size of $15^{\prime \prime} \times 25^{\prime \prime}$ that is consistent with ours.

In Fig. 3 we compare the column densities of FIRS 2 and Orion normalized to methanol. Normalization is required if one wants to compare the relative abundances of the different species in the warm gas. Methanol is commonly used to make this kind of normalization in hot cores (Öberg et al. 2011). Instead we could have used methyl cyanide for the normalization but we would have obtained essentially the same trend. As is clearly seen in Table 6 , it would simply produce an increase in all the ratios by a factor of 2 .

Most of the relative molecular abundances in FIRS 2 agree with those in Orion within a factor of 5 , which is quite good taking into account the uncertainty due to the unknown spatial structure of the molecular emission and opacity effects. Only a few molecules have abundances significantly different in both hot cores: $\mathrm{CH}_{3} \mathrm{OCHO}, \mathrm{CH}_{3} \mathrm{CH}_{2} \mathrm{CN}, \mathrm{HNCO}$, and $\mathrm{SO}_{2}$ have fractional abundances more than 10 times lower in FIRS 2 than in Orion. In the case of $\mathrm{CH}_{3} \mathrm{OCHO}$, we have not detected the ${ }^{13} \mathrm{C}$ isotopologues in FIRS 2 and our column density could be slightly underestimated. As discussed in Sect. 4.3, our estimate is accurate within a factor of 4 . Increasing the methyl formate by a factor of 4 would push the methyl formate back to the region within the two black lines in Fig. 3, although still in the lower end. In the case of HNCO, the lines of the main isotologue are blended with those of the ${ }^{13} \mathrm{C}$ isotopologue preventing us from a direct estimate of the line opacities. Looking at the top of the figure, there is a group of molecules that are more abundant in FIRS 2 by more than a factor of 4-5. These molecules are $\mathrm{CH}_{2} \mathrm{DOH}, \mathrm{D}_{2} \mathrm{CO}$, and $\mathrm{CH}_{3} \mathrm{CHO}$. As commented above, the value of $\mathrm{CH}_{3} \mathrm{CHO}$ in Orion is uncertain. However, the differences observed in $\mathrm{CH}_{2} \mathrm{DOH}$ and $\mathrm{D}_{2} \mathrm{CO}$ could be significant. In the following section, we discuss possible scenarios to explain these differences.

\subsection{Sequential formation of complex molecules in hot cores/corinos}

Although gas phase reactions can play a role, surface chemistry dominates the formation of COMs. The large sensitivity of surface chemistry on physical parameters, especially the dust temperature, can also explain the observed chemical differentiation within a hot core and among different hot cores. For example, Caselli et al. (1993) found that complex N-bearing species are more easily formed if the dust temperature $\left(T_{\text {dust }}\right)$ is about $40 \mathrm{~K}$ during the collapse phase. This is primarily due to the higher mobility of carbon and the shortage of hydrogen $(\mathrm{H}$, $\mathrm{H}_{2}$ ) on the grain surfaces. A similar argument has been used by Öberg et al. (2011) to explain the over-abundance of $\mathrm{CH}_{2 / 3}$-rich molecules in hot cores and corinos compared with molecular 


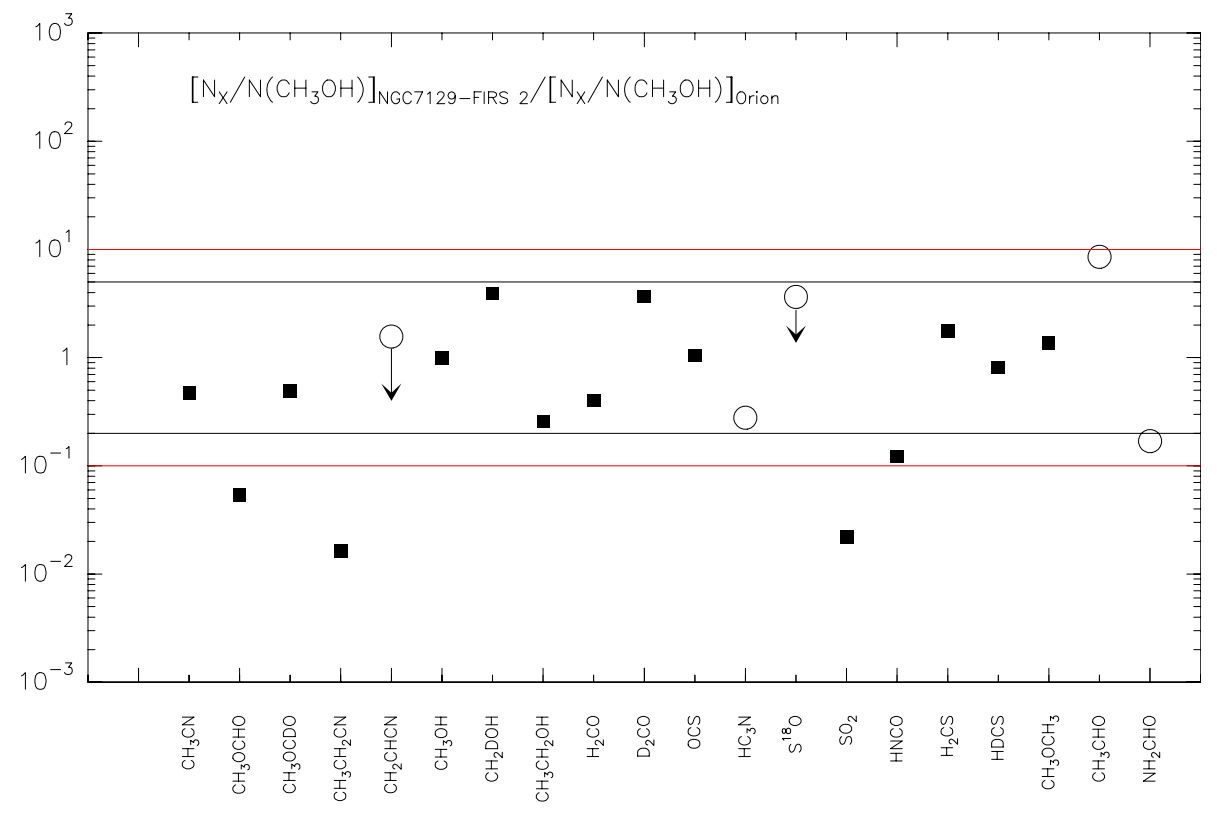

Fig. 3. Comparison of the molecular abundances in FIRS 2 and the Orion hot core (see Table 6). All the molecular abundances have been normalized to that of $\mathrm{CH}_{3} \mathrm{OH}$. Black horizontal lines indicate the loci of the FIRS 2 values that differ by less than a factor of 5 from those of Orion. Red lines indicate the same but for a factor of 10. Empty circles indicate doubtful values.

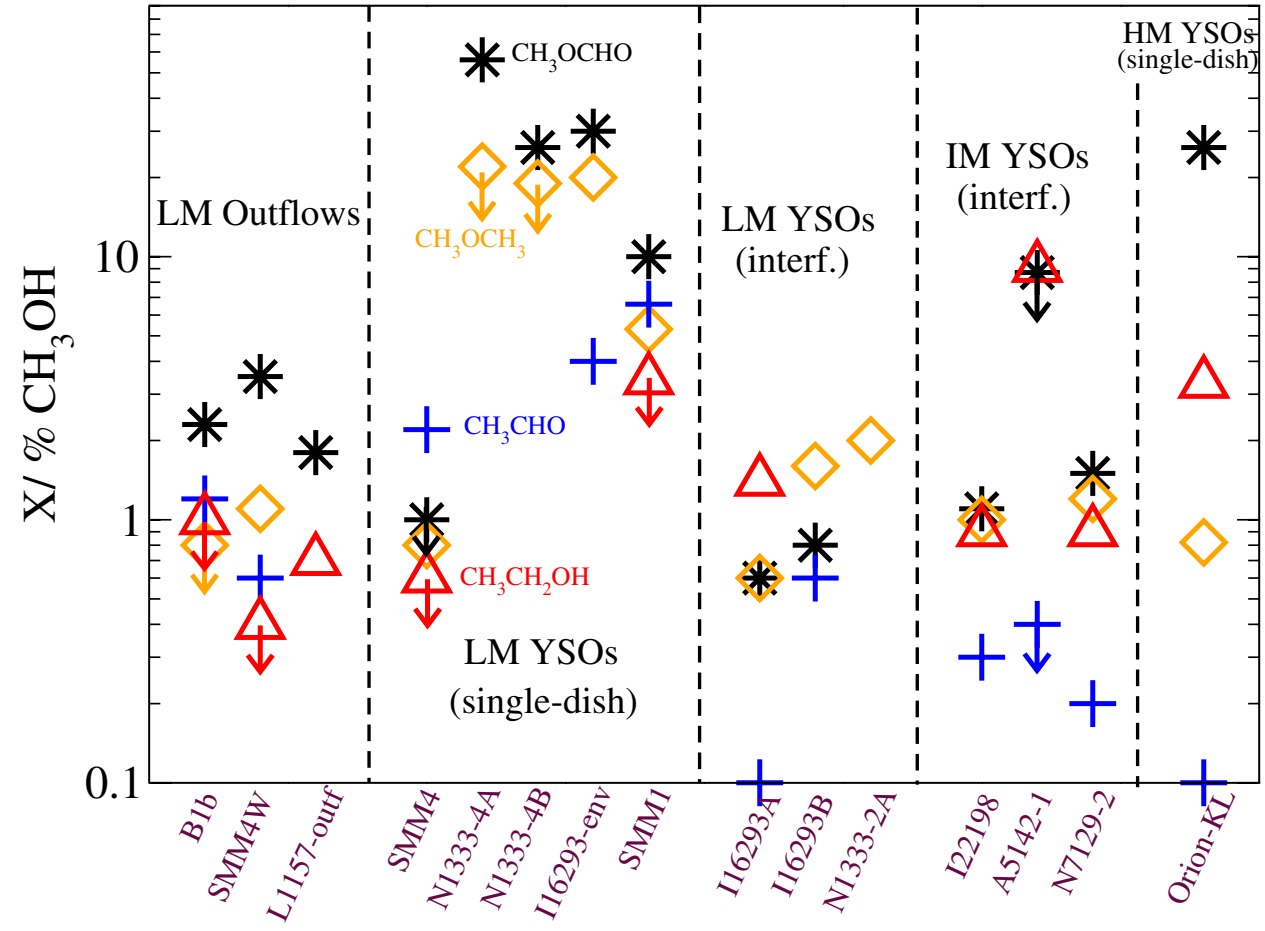

Fig. 4. Abundance of complex organic molecules relative to $\mathrm{CH}_{3} \mathrm{OH}$ for different types of sources, after Öberg et al. (2011): from left to right, low-mass outflows, low-mass YSOs observed with single-dish (tracing envelopes), low-mass YSOs observed with interferometers (tracing the inner envelope/disk at $~ 500 \mathrm{AU}$ spatial scale), intermediate-mass YSOs observed with interferometers (also at $500 \mathrm{AU}$ spatial scale, this work and Palau et al. 2011), and Orion (references in Table 6). Symbols with cold colors correspond to HCO-rich molecules (black: $\mathrm{CH}_{3} \mathrm{OCHO}$; blue: $\mathrm{CH}_{3} \mathrm{CHO}$ ), and symbols with warm colors correspond to $\mathrm{CH}_{3 / 2}$-rich molecules (orange: $\mathrm{CH}_{3} \mathrm{OCH}_{3}$; red: $\mathrm{CH}_{3} \mathrm{CH}_{2} \mathrm{OH}$ ). ouflows. Öberg et al. (2011) studied a number of cores associated with outflows, low-mass cores observed with single-dish telescopes (tracing envelopes at $~ 5000$ AU spatial scales), lowmass cores observed with interferometers (tracing warmer material at $\sim 500 \mathrm{AU}$ ), and an average of high-mass hot molecular cores from Bisschop et al. (2007), and they found that HCOrich molecules are in general more abundant than $\mathrm{CH}_{3 / 2}$-rich molecules in outflows and envelopes of low-mass YSOs, while the situation is reversed for low-mass inner envelopes/disks and high-mass YSOs. They proposed a sequential formation of complex molecules, starting with $\mathrm{HCO}$-rich molecules as long as $\mathrm{CO}$ ice is abundant, followed by $\mathrm{CH}_{3 / 2}$-rich molecules at higher ice temperatures.

In Fig. 4 we present an updated version of the figure by Öberg et al. (2011) complemented with abundances of three intermediate-mass hot cores observed with interferometers (at $~ 500$ AU scales, from this work and Palau et al. 2011), and the new Orion data. In the figure, symbols in warm colors (orange, red) correspond to $\mathrm{CH}_{3 / 2}$-rich molecules, while symbols in cold colors (blue/black) correspond to HCO-rich molecules. Our new results for the intermediate-mass hot core FIRS 2 are in line with previous measurements towards IM hot cores at diskscales ( $\sim 500 \mathrm{AU})$. Putting all these data together, we do not find a clear trend for the abundances of the $\mathrm{CH}_{3 / 2}$-rich and $\mathrm{HCO}$ rich molecules with the stellar mass. Considering only interferometric observations, the $\mathrm{CH}_{3} \mathrm{CHO}$ abundance seems to decrease with the stellar mass. However, the abundance of methyl formate, the only other HCO-rich molecule considered, is larger in Orion than in IM hot cores and hot corinos. Orion is the only source in which we have been able to use the ${ }^{13} \mathrm{C}$ isotopologue to derive the methyl formate abundance. If the methyl formate lines were typically optically thick in hot cores/corinos, 
this would have produced an underestimate of the $\mathrm{CH}_{3} \mathrm{OCHO}$ abundance in the rest of objects. Using the upper limit to the emission of the ${ }^{13} \mathrm{C}$ isotopologue, we have estimated that the methyl formate abundance in FIRS 2 is accurate within a factor of 4 . Even considering an uncertainty of a factor of 4 in the $\mathrm{CH}_{3} \mathrm{OCHO}$ abundances towards low-mass and IM YSOs, the $\left[\mathrm{CH}_{3} \mathrm{OCHO}\right] /\left[\mathrm{CH}_{3} \mathrm{OH}\right]$ ratio would be larger in Orion than in these objects. In addition, the $\left[\mathrm{CH}_{3} \mathrm{OCHO}\right] /\left[\mathrm{CH}_{3} \mathrm{OH}\right]$ ratio seems to increase from the low-mass to the IM sample. We can only compare two HCO-rich molecules in Fig. 4. Therefore we cannot know if methyl formate is a special case (see also Taquet et al. 2012). Further observations in IM and massive hot cores are required to have a deeper insight into the chemistry of this molecule and other HCO-rich species.

We have yet to discuss the deuterium fractions in FIRS 2 and Orion. Deuterated species whose deuteration requires surface chemistry, such as $\mathrm{D}_{2} \mathrm{CO}$, present higher abundances in the warm regions associated with low-mass protostars. Loinard et al. (2003) searched for the doubly deuterated form of formaldehyde $\left(\mathrm{D}_{2} \mathrm{CO}\right)$ in a large sample of young stellar objects; $\mathrm{D}_{2} \mathrm{CO}$ was detected in all low-mass protostars, with $\left[\mathrm{D}_{2} \mathrm{CO}\right] /\left[\mathrm{H}_{2} \mathrm{CO}\right]$ ratios of $2-40 \%$. On the other hand, no detection was obtained towards massive protostars (where $\left[\mathrm{D}_{2} \mathrm{CO}\right] /\left[\mathrm{H}_{2} \mathrm{CO}\right]<0.5 \%$ ). This is consistent with the value reported by Turner (1990) in Orion. If the hot cores associated with massive stars are older and/or significantly denser than those surrounding low-mass objects, gas phase chemistry could have had the time to reset the deuterium fractions to values close to the cosmic $\mathrm{D} / \mathrm{H}$ ratio. The longer time of the evaporated molecules in gas phase would also affect the abundances of the COMs that can be formed in warm gas-phase chemistry (e.g., $\mathrm{CH}_{2} \mathrm{CHCN}$ ).

An alternative explanation could be that the temperature of the envelope material accreting onto the high-mass protostars is larger than $30 \mathrm{~K}$ (Fontani et al. 2002). In this case the deuterium fractionation efficiency in the gas phase is strongly reduced before the hot core phase as the standard $\mathrm{H}_{2} \mathrm{D}^{+}$route $\left(\mathrm{H}_{3}^{+}+\mathrm{HD} \rightarrow\right.$ $\mathrm{H}_{2} \mathrm{D}^{+}+\mathrm{H}_{2}$ ) also starts to proceed from left to right. Moreover, at $T_{\text {dust }}>30 \mathrm{~K}$ there is no way that deuteration can be enhanced on the grain surfaces. The reason is that $H$ and $D$ are easily evaporated before reacting on the surface (see e.g. Cazaux et al. 2011). The higher temperature during the collapse would also affect the formation of COMs in grain mantles. As CO does not efficiently freeze out at these values of $T_{\text {dust }}$, the formation of HCO-rich molecules would be strongly diminished too. As commented above, methyl formate could be an exception to this rule.

\subsection{Carbon budget}

We have used the column densities shown in Table 3 to estimate the carbon gas phase budget in the FIRS 2 hot core. Apart from $\mathrm{CO}$ and its isotopologues, most of the carbon is locked in methanol with a total column density of $\sim 3.4 \times 10^{20} \mathrm{~cm}^{-2}$ in the compact component of the hot core. Taking into account the rest of molecules, the carbon column density reaches to $\sim 4 \times 10^{20} \mathrm{~cm}^{-2}$ in this small region. One problem in deriving absolute abundances is that we do not know the total column density of gas in the inner $R<0.05^{\prime \prime}$ region. Based on higher angular resolution observations, FU05 derived a size of $0.72^{\prime \prime} \times 0.52^{\prime \prime}$ for the continuum source that account for $75 \%$ of the continuum emission and a point source that contributes to $25 \%$ of the flux. Adopting this value for the fraction of continuum flux coming from the compact component of the hot core and a total gas mass of $0.46 M_{\odot}$ (see Sect. 6.1), the total column density of molecular hydrogen would be $2 \times 10^{25} \mathrm{~cm}^{-2}$ and the fractional abundance of the carbon locked in COMs relative to $\mathrm{H}$ nuclei, $\sim 1.0 \times 10^{-5}$. This value is 36 times lower than the solar value of the carbon abundance $\left(\mathrm{C} / \mathrm{H} \sim 3.6 \times 10^{-4}\right.$; Anders \& Grevesse 1989) and suggests that methanol and COMs (at least those considered in this paper) are not the main reservoirs of carbon in hot cores.

It is also useful to compare methanol with $\mathrm{C}^{18} \mathrm{O}$. Again we have the problem of the unknown spatial distribution of the $\mathrm{C}^{18} \mathrm{O}$ emission within our interferometric beam. We can derive a lower limit to the $\mathrm{C}^{18} \mathrm{O}$ abundance assuming that the emission is optically thin and fills the beam. Adopting $T_{\text {rot }}=400 \mathrm{~K}$, we derive $N\left(\mathrm{C}^{18} \mathrm{O}\right)=1.5 \times 10^{17} \mathrm{~cm}^{-2}$ and $N(\mathrm{CO})=9.4 \times 10^{19} \mathrm{~cm}^{-2}$. This solution, however, is not compatible with the non-detection of the $\mathrm{C}^{18} \mathrm{O} 9 \rightarrow 8$ line by Fuente et al. (2012) and we cannot reconcile the PdBI and Herschel results by lowering the temperature down to $T_{\text {rot }}=200 \mathrm{~K}$. The optically thick solution seems more reasonable. Assuming again that the gas temperature is $400 \mathrm{~K}$ and that the $\mathrm{C}^{18} \mathrm{O}$ emission is optically thick $\left(T_{\mathrm{B}} \approx T_{\text {rot }}\right)$, we derive an effective diameter of $0.15^{\prime \prime}$ for the $\mathrm{C}^{18} \mathrm{O}$ emitting region. In this case, our results are compatible with the Herschel upper limit to the $\mathrm{C}^{18} \mathrm{O} 9 \rightarrow 8$ line and the column density of $\mathrm{CO}$ would be $>10^{22} \mathrm{~cm}^{-2}$ in the compact component of the hot core.

\section{Conclusions}

The protostar FIRS 2 is probably the youngest IM protostar studied thus far and, as such, is an excellent template on which to base interpretations of other IM and massive star forming regions. The interferometric observations presented in this paper proved that this IM protostar hosts a hot core extraordinarily rich in complex molecules. The gas kinetic temperature as measured with methyl cyanide, is around $400 \mathrm{~K}$, similar to that of the Orion hot core and higher than typical rotation temperatures in hot corinos. A detailed comparison of the chemistry of FIRS 2 with Orion shows that the fractional abundances of most molecules relative to that of $\mathrm{CH}_{3} \mathrm{OH}$ agree within a factor of 5, which is reasonable taking into account the uncertainties of our column density estimates. Only $\mathrm{CH}_{3} \mathrm{CH}_{2} \mathrm{CN}$, $\mathrm{HNCO}$, $\mathrm{SO}_{2}, \mathrm{CH}_{2} \mathrm{DOH}, \mathrm{D}_{2} \mathrm{CO}$, and $\mathrm{CH}_{3} \mathrm{CHO}$ present a significant disagreement; $\mathrm{CH}_{3} \mathrm{CH}_{2} \mathrm{CN}, \mathrm{HNCO}$, and $\mathrm{SO}_{2}$ are more abundant in Orion, and $\mathrm{CH}_{2} \mathrm{DOH}, \mathrm{D}_{2} \mathrm{CO}$, and $\mathrm{CH}_{3} \mathrm{CHO}$, in FIRS 2. Since the physical conditions are similar in both hot cores, only different initial conditions (warmer pre-collapse and collapse phase in the case of Orion) and/or different crossing time of the gas in the hot core can explain this behavior. FIRS 2 is a YSO in which most of the accreting envelope still maintains an overall temperature lower than $30 \mathrm{~K}$, allowing deuteration to proceed unhindered, while complex N-bearing molecules like $\mathrm{CH}_{3} \mathrm{CH}_{2} \mathrm{CN}$ are not efficiently formed, unlike the Orion hot core.

Acknowledgements. We thank the Spanish MINECO for funding support from grants CSD2009-00038, AYA2009-07304, and AYA2012-32032. D.J. is supported by the National Research Council of Canada and by a Natural Sciences and Engineering Research Council of Canada (NSERC) Discovery Grant. A.P. is supported by the Spanish MICINN grant AYA2011-30228-C03-02 (co-funded with FEDER funds), and by the AGAUR grant 2009SGR1172 (Catalonia).

\section{References}

Adande, G. R., Woolf, N. J., \& Ziurys, L. M. 2013, Astrobiology, 13, 439 Alonso-Albi, T., Fuente, A., Crimier, N., et al. 2010, A\&A, 518, A52 Anders, E., \& Grevesse, N. 1989, Geochim. Cosmochim. Acta, 53, 197 Bell, T. A., Cernicharo, J., Viti, S., et al. 2014, A\&A, 564, A114 Belloche, A., Müller, H. S. P., Menten, K. M., Schilke, P., \& Comito, C. 2013, A\&A, 559, A47 
Beltrán, M. T., Codella, C., Viti, S., Neri, R., \& Cesaroni, R. 2009, ApJ, 690, L93

Beuther, H., Zhang, Q., Greenhill, L. J., et al. 2005, ApJ, 632, 355

Beuther, H., Zhang, Q., Reid, M. J., et al. 2006, ApJ, 636, 323

Bisschop, S. E., Jørgensen, J. K., Bourke, T. L., Bottinelli, S., \& van Dishoeck, E. F. 2008, A\&A, 488, 959

Blake, G. A., Sutton, E. C., Masson, C. R., \& Phillips, T. G. 1987, ApJ, 315, 621

Bottinelli, S., Ceccarelli, C., Neri, R., et al. 2004a, ApJ, 617, L69

Bottinelli, S., Ceccarelli, C., Lefloch, B., et al. 2004b, ApJ, 615, 354

Bottinelli, S., Ceccarelli, C., Williams, J. P., \& Lefloch, B. 2007, A\&A, 463, 601

Brouillet, N., Despois, D., Baudry, A., et al. 2013, A\&A, 550, A46

Carvajal, M., Margulès, L., Tercero, B., et al. 2009, A\&A, 500, 1109

Cazaux, S., Tielens, A. G. G. M., Ceccarelli, C., et al. 2003, ApJ, 593, L51

Cazaux, S., Caselli, P., \& Spaans, M. 2011, ApJ, 741, L34

Cernicharo, J. 2012, in Proc. Eur. Conf. Laboratory Astrophysics, EAS Pub. Ser., eds. C. Stehlé, C. Joblin, \& L. d'Hendecourt

Cernicharo, J., Kahane, C., Guelin, M., \& Gómez-Gonzalez, J. 1988, A\&A, 189, L1

Cernicharo, J., Tercero, B., Fuente, A., et al. 2013, ApJ, 771, L10

Chin, Y.-N., Henkel, C., Whiteoak, J. B., Langer, N., \& Churchwell, E. B. 1995, VizieR Online Data Catalog

Comito, C., Schilke, P., Phillips, T. G., et al. 2005, ApJS, 156, 127

Daly, A. M., Bermúdez, C., López, A., et al. 2013, ApJ, 768, 81

Demyk, K., Bottinelli, S., Caux, E., et al. 2010, A\&A, 517, A17

Esplugues, G. B., Tercero, B., Cernicharo, J., et al. 2013a, A\&A, 556, A143

Esplugues, G. B., Cernicharo, J., Viti, S., et al. 2013b, A\&A, 559, A51

Fich, M., Johnstone, D., van Kempen, T. A., et al. 2010, A\&A, 518, L86

Fuente, A., Neri, R., Martín-Pintado, J., et al. 2001, A\&A, 366, 873

Fuente, A., Neri, R., \& Caselli, P. 2005a, A\&A, 444, 481 (FU05)

Fuente, A., Rizzo, J. R., Caselli, P., Bachiller, R., \& Henkel, C. 2005b, A\&A, 433,535

Fuente, A., Castro-Carrizo, A., Alonso-Albi, T., et al. 2009, A\&A, 507, 1475

Fuente, A., Caselli, P., McCoey, C., et al. 2012, A\&A, 540, A75

Halfen, D. T., Apponi, A. J., Woolf, N., Polt, R., \& Ziurys, L. M. 2006, ApJ, 639, 237

Haykal, I., Carvajal, M., Tercero, B., et al. 2014, A\&A, in press, DOI: $10.1051 / 0004-6361 / 201322937$

Hollis, J. M., Lovas, F. J., \& Jewell, P. R. 2000, ApJ, 540, L107

Johnstone, D., Fich, M., McCoey, C., et al. 2010, A\&A, 521, L41

Kahane, C., Ceccarelli, C., Faure, A., \& Caux, E. 2013, ApJ, 763, L38

Kolesniková, L., Tercero, B., Cernicharo, J., et al. 2014, ApJ, 784, L7
Jørgensen, J. K., Bourke, T. L., Myers, P. C., et al. 2005, ApJ, 632, 973 Jørgensen, J. K., Bourke, T. L., Myers, P. C., et al. 2007, ApJ, 659, 479 Loinard, L., Castets, A., Ceccarelli, C., et al. 2003, SFChem 2002: Chemistry as a Diagnostic of Star Formation, 351

López, A., Tercero, B., Kisiel, Z., et al. 2007, A\&A, submitted

Marcelino, N., Cernicharo, J., Tercero, B., \& Roueff, E. 2009, ApJ, 690, L27

Margulès, L., Huet, T. R., Demaison, J., et al. 2010, ApJ, 714, 1120

Milam, S. N., Savage, C., Brewster, M. A., Ziurys, L. M., \& Wyckoff, S. 2005, ApJ, 634, 1126

Motiyenko, R. A., Tercero, B., Cernicharo, J., \& Margulès, L. 2012, A\&A, 548, A71

Müller, H. S. P., Thorwirth, S., Roth, D. A., \& Winnewisser, G. 2001, A\&A, 370, L49

Müller, H. S. P., Schlöder, F., Stutzki, J., \& Winnewisser, G. 2005, J. Mol. Struct., 742,215

Neill, J. L., Crockett, N. R., Bergin, E. A., Pearson, J. C., \& Xu, L.-H. 2013 ApJ, 777, 85

Neri, R., Fuente, A., Ceccarelli, C., et al. 2007, A\&A, 468, L33

Palau, A., Fuente, A., Girart, J. M., et al. 2011, ApJ, 743, L32

Palau, A., Fuente, A., Girart, J. M., et al. 2013, ApJ, 762, 120

Parise, B., Castets, A., Herbst, E., et al. 2004, A\&A, 416, 159

Peng, T.-C., Despois, D., Brouillet, N., et al. 2013, A\&A, 554, A78

Pickett, H. M. 1991, J. Molec. Spectr., 148, 371

Pickett, H. M., Poynter, R. L., Cohen, E. A., et al. 1998, J. Quant. Spectr. Rad. Trans., 60, 883

Pineda, J. E., Maury, A. J., Fuller, G. A., et al. 2012, A\&A, 544, L7

Öberg, K. I., van der Marel, N., Kristensen, L. E., \& van Dishoeck, E. F. 2011, ApJ, 740, 14

Requena-Torres, M. A., Martín-Pintado, J., Martín, S., \& Morris, M. R. 2008, ApJ, 672, 352

Sánchez-Monge, Á., Palau, A., Estalella, R., et al. 2010, ApJ, 721, L107

Shevchenko, V. S., \& Yakubov, S. D. 1989, Sov. Ast, 33, 370

Taquet, V., Ceccarelli, C., \& Kahane, C. 2012, A\&A, 538, A42

Tercero, B., Cernicharo, J., Pardo, J. R., \& Goicoechea, J. R. 2010, A\&A, 517, A96

Tercero, B., Vincent, L., Cernicharo, J., Viti, S., \& Marcelino, N. 2011, A\&A, 528, A26

Tercero, B., Kleiner, I., Cernicharo, J., et al. 2013, ApJ, 770, L13

Turner, B. E. 1990, ApJ, 362, L29

Turner, B. E. 1991, ApJS, 76, 617

Vastel, C., Phillips, T. G., Ceccarelli, C., \& Pearson, J. 2003, ApJ, 593, L97

Zapata, L. A., Loinard, L., Rodríguez, L. F., et al. 2013, ApJ, 764, L14 


\section{Appendix A}
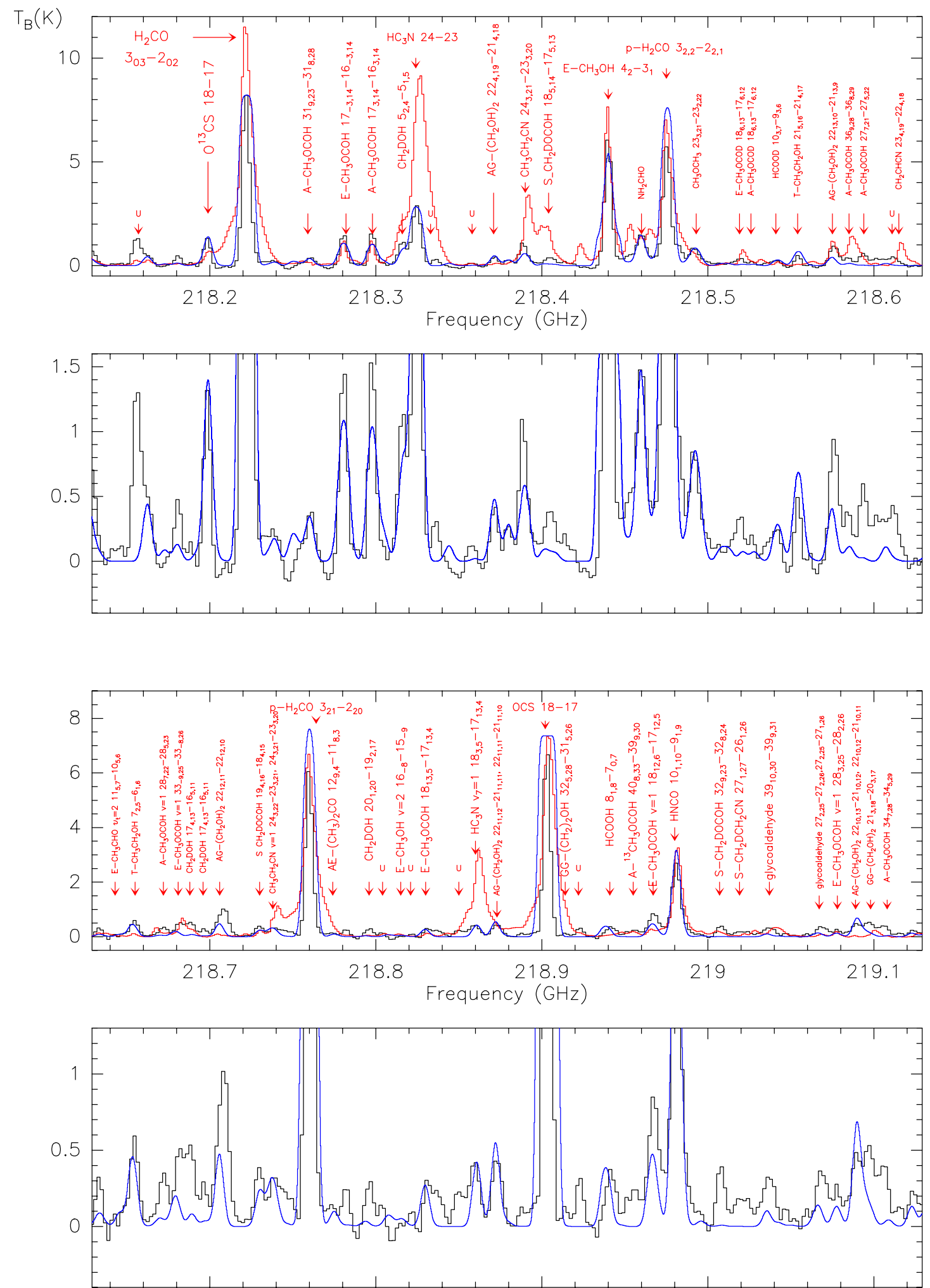

Fig. A.1. Comparison of the interferometric spectra towards NGC 7129 - FIRS 2 (black line) with the single-dish spectrum of Orion KL (red line). The Orion spectra were provided by B. Tercero and J. Cernicharo and multiplied by a factor 0.43 for an easier comparison. The spectrum was observed towards: $5^{\mathrm{h}} 35^{\mathrm{m}} 14.5,-5^{\circ} 22^{\prime} 30^{\prime} 0(\mathrm{~J} 2000)$ and is centered at a velocity of $7 \mathrm{~km} \mathrm{~s}^{-1}$. Lines are labeled in red. When several lines are blended, only the most intense ones are indicated. The spectra synthesized with the parameters shown in Table 3 are in blue. The intensity scale is brightness temperature. 
A. Fuente et al.: The hot core towards the intermediate-mass protostar NGC 7129 FIRS 2


Fig. A.1. continued. 
A\&A 568, A65 (2014)
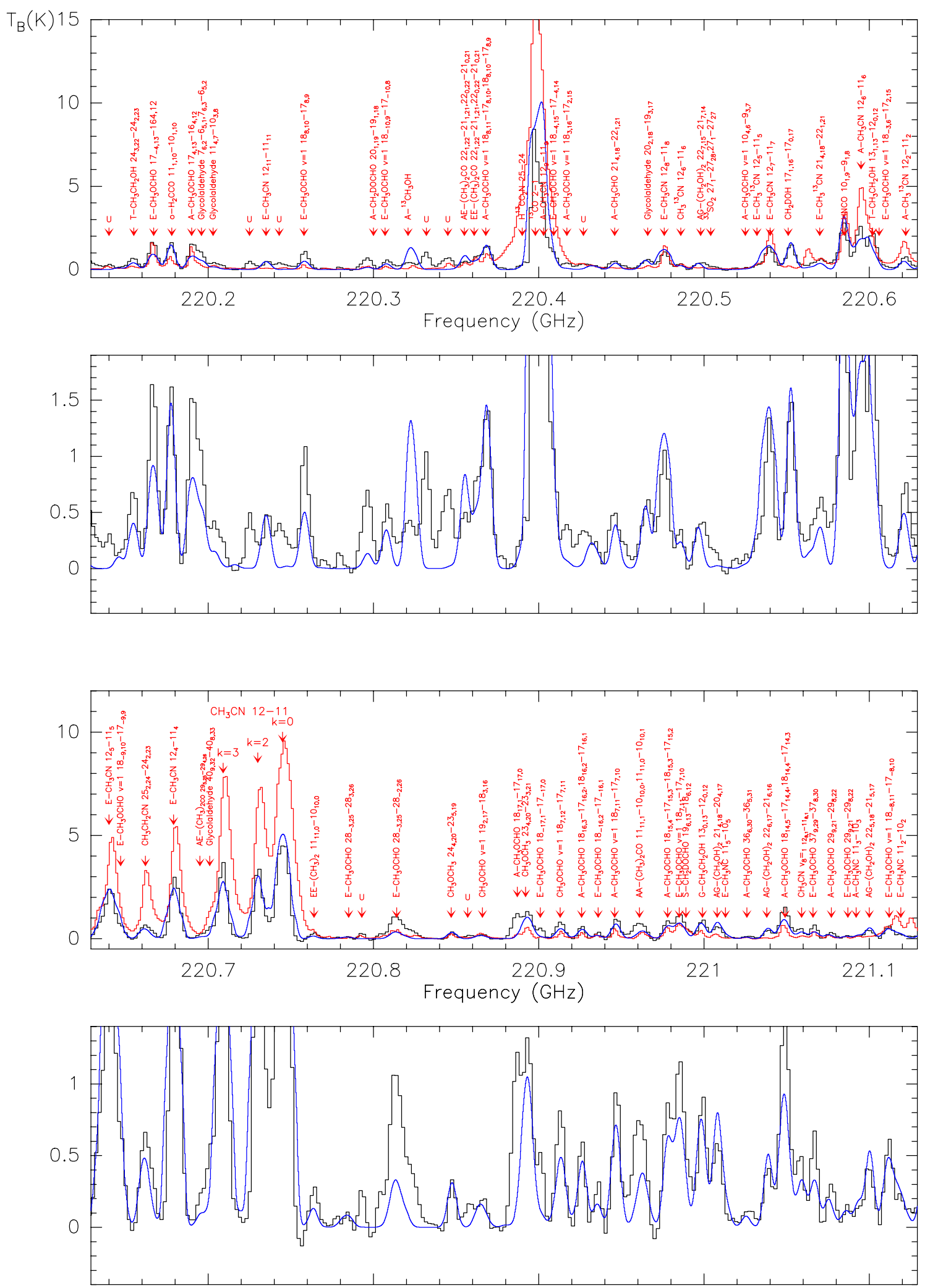

Fig. A.1. continued. 
A. Fuente et al.: The hot core towards the intermediate-mass protostar NGC 7129 FIRS 2


Fig. A.1. continued.
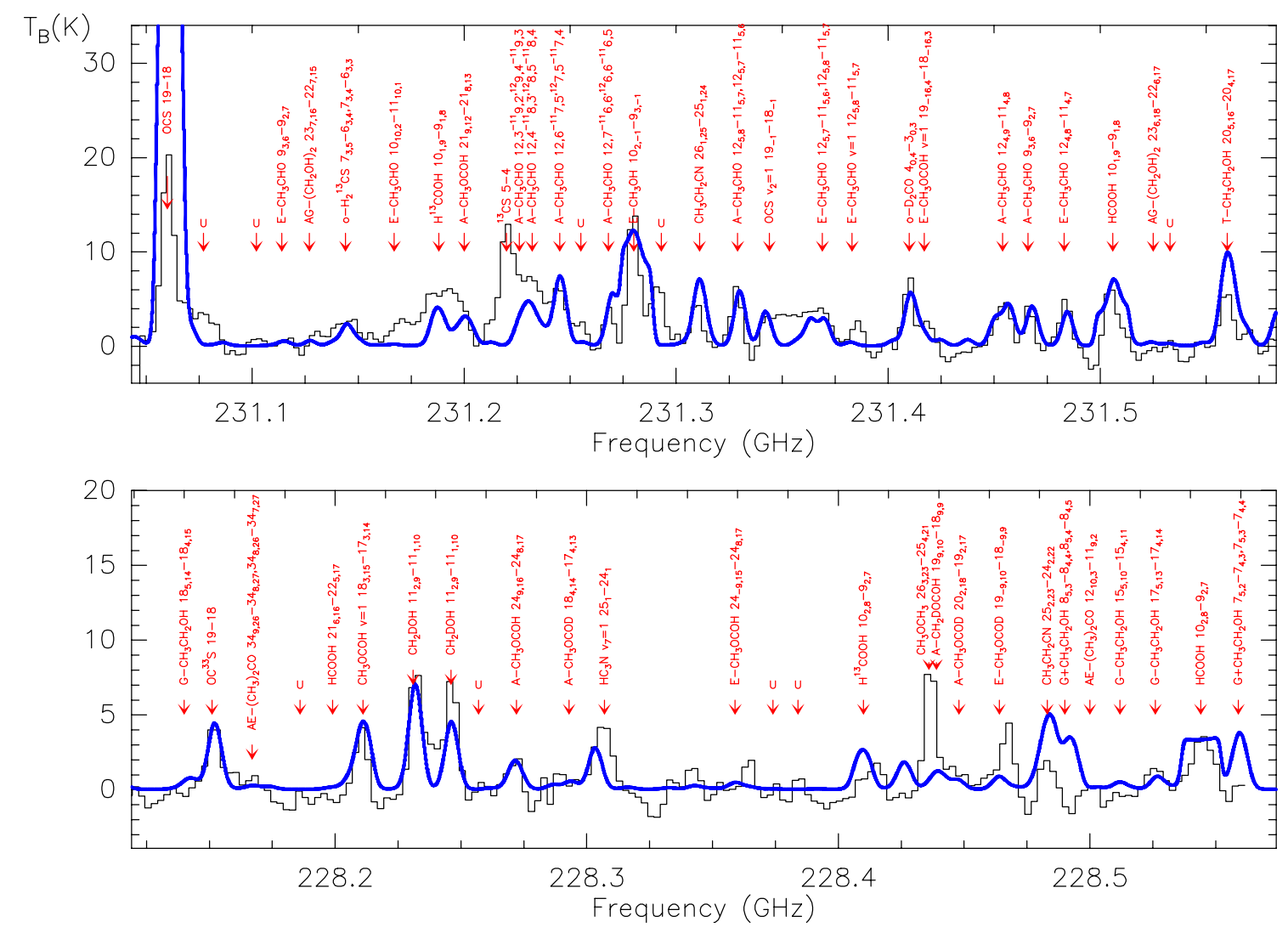

Fig. A.2. In blue, our synthetic spectrum superposed on the interferometric spectra published by FU05. 
Table A.1. Line identifications.

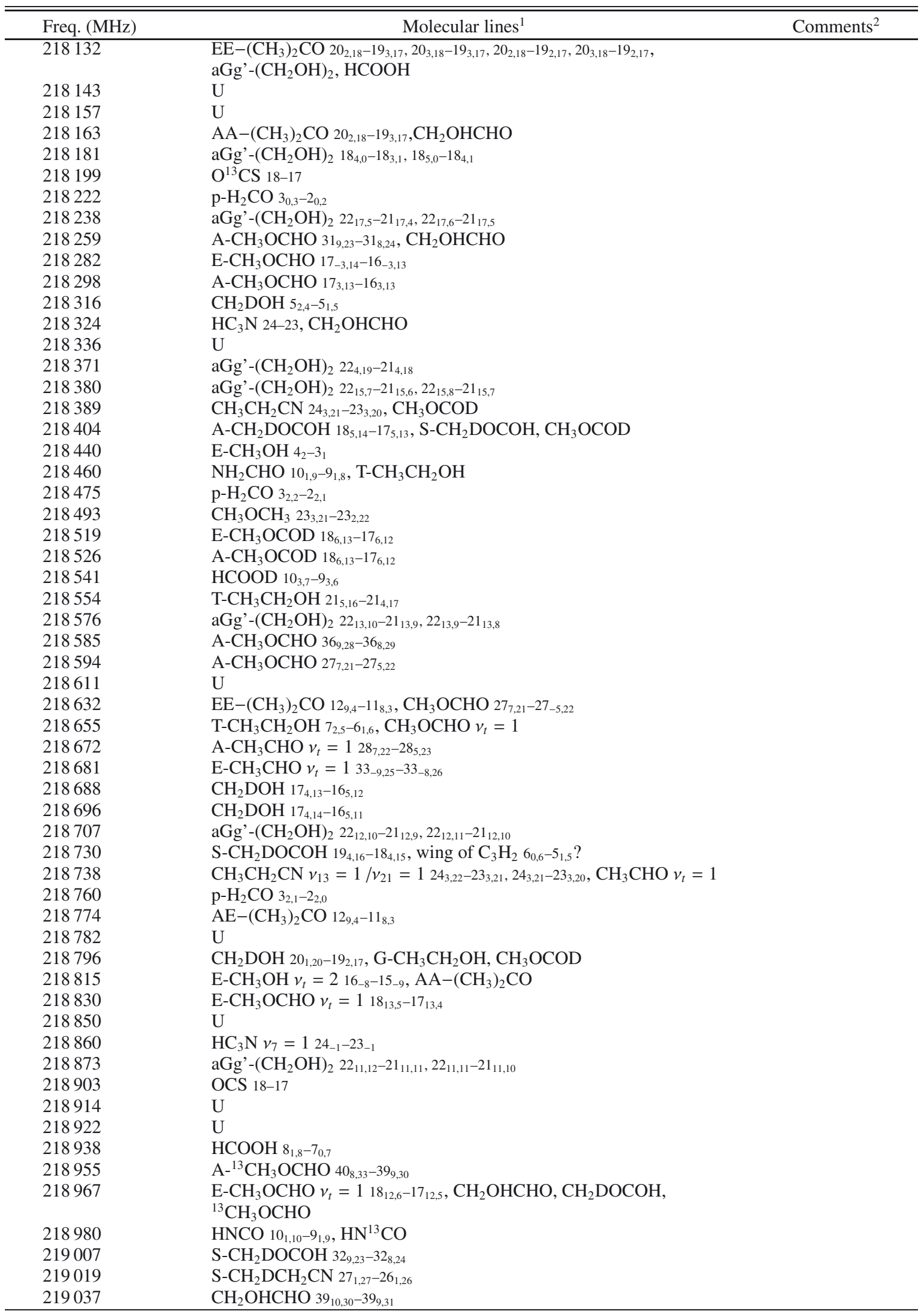

Notes. ${ }^{(1)}$ Lines are ordered from major to minor contribution to the total integrated flux according to our model. (2) The lines in this column correspond to doubtful identifications. 
Table A.1. continued.




Table A.1. continued.




Table A.1. continued.




Table A.1. continued.

\begin{tabular}{|c|c|c|}
\hline Freq. (MHz) & Molecular lines $^{1}$ & Comments $^{2}$ \\
\hline 221534 & $\mathrm{U}$ & \\
\hline 221547 & $\mathrm{U}$ & \\
\hline 221560 & A-CH ${ }_{2}$ DOCOH $7_{6,2}-66_{5,1}, 7_{6,1}-6_{5,2}, \mathrm{~S}-\mathrm{CH}_{2}$ DOCOH $21_{3,18}-20_{4,17}, 50_{9,41}-50_{8,42}$ & \\
\hline 221566 & $\mathrm{U}$ & \\
\hline 221582 & aGg' $-\left(\mathrm{CH}_{2} \mathrm{OH}\right)_{2} \quad 18_{4,15}-17_{3,14}$ & \\
\hline 221597 & $\mathrm{U}$ & \\
\hline 221605 & $\mathrm{U}$ & \\
\hline 221627 & $\mathrm{CH}_{3} \mathrm{CN} v_{8}=112_{1,2}-11_{-1,2}$ & \\
\hline 221649 & E- $\mathrm{CH}_{3} \mathrm{OCHO} 18_{-10,8}-17_{-10,7}$ & \\
\hline 221660 & A- $\mathrm{CH}_{3} \mathrm{OCHO} 18_{10,9}-17_{10,8}, 18_{10,8}-17_{10,7}$, E- $\mathrm{CH}_{3} \mathrm{OCHO} 18_{4,15}-17_{4,14}$ & \\
\hline
\end{tabular}

Table A.2. Line identifications (FU05).

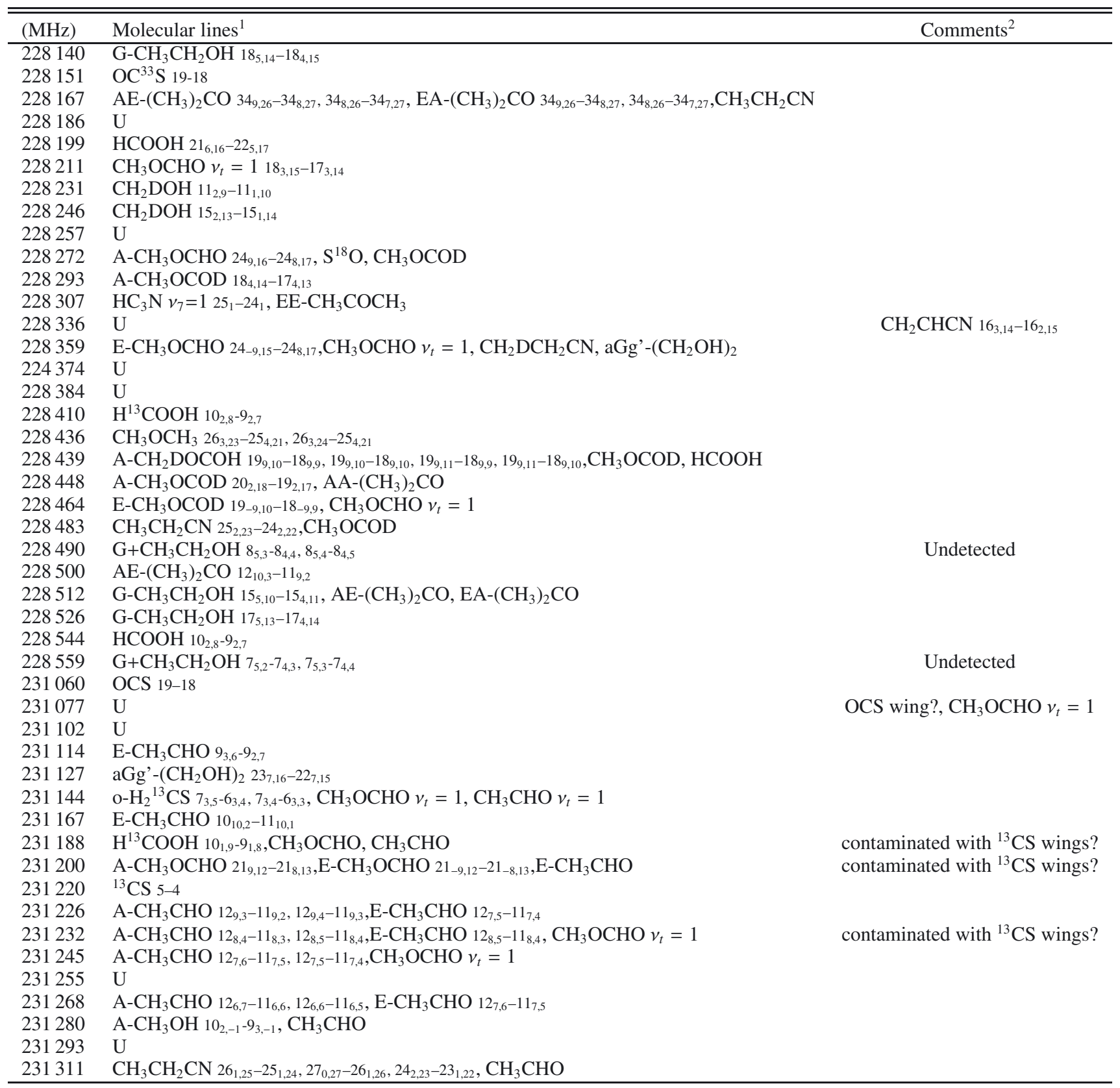

Notes. ${ }^{(1)}$ Lines are ordered from major to minor contribution to the total integrated flux according with our model. ${ }^{(2)}$ The lines in this column correspond to doubtful identifications. 
Table A.2. continued.

\begin{tabular}{|c|c|c|}
\hline$(\mathrm{MHz})$ & Molecular lines ${ }^{1}$ & Comments $^{2}$ \\
\hline 231329 & $\mathrm{~A}-\mathrm{CH}_{3} \mathrm{CHO} 12_{5,8}-11_{5,7}, 12_{5,7}-11_{5,6}$ & \\
\hline 231344 & OCS $v_{2}=119_{-1}-18_{-1}$ & \\
\hline 231369 & E- $\mathrm{CH}_{3} \mathrm{CHO}{ }_{125,7}-11_{5,6}, 12_{5,8}-11_{5,7}$ & \\
\hline 231383 & E- $\mathrm{CH}_{3} \mathrm{CHO} v=1 \quad 12_{5,8}-11_{5,7}$ & \\
\hline 231410 & $\mathrm{o}-\mathrm{D}_{2} \mathrm{CO} 4_{0,4}-3_{0,3}$ & \\
\hline 231417 & $\mathrm{E}-\mathrm{CH}_{3} \mathrm{COOH} v=11_{-16,4}-18_{-16,3}$ & \\
\hline 231454 & $\mathrm{~A}-\mathrm{CH}_{3} \mathrm{CHO}{ }_{12} 2_{4,9}-11_{4,8}$ & \\
\hline 231466 & $\mathrm{~A}-\mathrm{CH}_{3} \mathrm{CHO} 9_{3,6}-9_{2,7}$ & \\
\hline 231483 & E- $\mathrm{CH}_{3} \mathrm{CHO} 12_{4,8}-11_{4,7}$ & \\
\hline 231506 & $\mathrm{HCOOH} 10_{1,9}-9_{1,8}, \mathrm{CH}_{3} \mathrm{CHO}$ & \\
\hline $231525 ?$ & aGg'-( $\left.\mathrm{CH}_{2} \mathrm{OH}\right)_{2} 23_{6,18}-22_{6,17}$ & \\
\hline $231533 ?$ & $\mathrm{U}$ & \\
\hline 231560 & $\mathrm{~T}-\mathrm{CH}_{3} \mathrm{CH}_{2} \mathrm{OH} 20_{5,16}-20_{4,17}$ & \\
\hline
\end{tabular}

Table A.3. $\mathrm{CH}_{3} \mathrm{CN} v=0$ line parameters.

\begin{tabular}{|c|c|c|c|c|c|c|c|c|c|}
\hline$N^{1}$ & 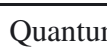 & numbers: up-low ${ }^{2}$ & $v(\mathrm{MHz})$ & $\overline{E_{u}(\mathrm{~K})^{3}}$ & $A_{\mathrm{ul}}\left(\mathrm{s}^{-1}\right)^{4}$ & $S_{i j}^{5}$ & $g u^{6}$ & $W\left(\mathrm{~K} \mathrm{~km} \mathrm{~s}^{-1}\right)^{7}$ & \\
\hline 1 & 1211 & $11 \quad 11$ & 220235.047 & 923.4 & $1.469 \mathrm{E}-04$ & 1.92 & 25 & $1.95(1.95)$ & \multirow{10}{*}{ Blended with $\mathrm{A}-{ }^{13} \mathrm{CH}_{3} 3 \mathrm{OH}$} \\
\hline 2 & 1210 & 1110 & 220323.643 & 774.0 & $2.813 \mathrm{E}-04$ & 3.67 & 25 & $4.73(1.38)$ & \\
\hline 3 & 128 & 118 & 220475.814 & 517.6 & $5.125 \mathrm{E}-04$ & 6.67 & 25 & $7.57(1.17)$ & \\
\hline 4 & 127 & 117 & 220539.328 & 410.6 & $6.091 \mathrm{E}-04$ & 7.92 & 25 & $10.18(6.0)$ & \\
\hline 5 & 124 & 114 & 220679.287 & 175.1 & $8.223 \mathrm{E}-04$ & 10.7 & 25 & $27.05(10.63)$ & \\
\hline 6 & 122 & 112 & 220730.259 & 89.4 & $9.000 \mathrm{E}-04$ & 11.7 & 25 & $29.82(6.74)$ & \\
\hline 7 & 121 & 111 & 220743.008 & 68.0 & $9.195 \mathrm{E}-04$ & 11.9 & 25 & $58.83(7.66)$ & \\
\hline 7 & 120 & 110 & 220747.259 & 68.9 & $9.259 \mathrm{E}-04$ & 12.0 & 25 & & \\
\hline 8 & 126 & 116 & 220594.426 & 325.9 & $3.465 \mathrm{E}-04$ & 9.00 & 50 & $19.37(6.83)$ & \\
\hline 9 & 123 & 113 & 220709.015 & 133.2 & $4.338 \mathrm{E}-04$ & 11. & 50 & $31.35(10.23)$ & \\
\hline
\end{tabular}

Notes. Exponential notation: $1.469 \mathrm{E}-04=1.469 \times 10^{-4}$. ${ }^{(1)}$ Index to enumerate the line features in the spectrum (blended lines share the same index). ${ }^{(2)}$ Quantum numbers of the upper and lower levels of the transitions. ${ }^{(3)}$ Energy of the upper level. ${ }^{(4)}$ Einstein coefficient of spontaneous emission. ${ }^{(5)}$ Line strength defined as $\left|\mu_{i j}\right|^{2}=S_{i j} \mu^{2}$ where $\mu$ is the dipole moment. ${ }^{(6)}$ Degeneracy of the upper level. ${ }^{(7)}$ Velocity integrated intensity. 
Table A.4. $\mathrm{CH}_{3} \mathrm{CN} v_{8}=1\left(E_{\mathrm{vib}}=525.17 \mathrm{~K}\right)$ line parameters.

\begin{tabular}{|c|c|c|c|c|c|c|c|c|c|c|c|c|c|c|c|}
\hline$N^{1}$ & & dantu & $\mathrm{mn}$ & numb & bers & up & -low & & $v(\mathrm{MHz})$ & $E_{R_{u}}(\mathrm{~K})^{3}$ & $A_{\mathrm{ul}}\left(\mathrm{s}^{-1}\right)^{4}$ & $S_{i j}^{5}$ & $g u^{6}$ & $W\left(\mathrm{~K} \mathrm{~km} \mathrm{~s}^{-1}\right)^{7}$ & \\
\hline 1 & 12 & 8 & 1 & 12 & 11 & 8 & 1 & 11 & 221059.437 & 637.3 & $1.906 \mathrm{E}-03$ & 24.6 & 25 & $3.63(1.57)$ & \\
\hline 1 & 12 & 8 & 1 & 13 & 11 & 8 & 1 & 12 & 221059.797 & 637.3 & $1.923 \mathrm{E}-03$ & 26.8 & 27 & & \\
\hline 1 & 12 & 8 & 1 & 11 & 11 & 8 & 1 & 10 & 221059.828 & 637.3 & $1.904 \mathrm{E}-03$ & 22.6 & 23 & & \\
\hline 2 & 12 & -1 & 2 & 11 & 11 & 1 & 2 & 10 & 221199.140 & 68.1 & $1.705 \mathrm{E}-03$ & 20.2 & 23 & 17.62(8.89) & Blended with $\mathrm{CH}_{3} \mathrm{OCH}_{3}$ \\
\hline 2 & 12 & -1 & 2 & 12 & 11 & 1 & 2 & 11 & 221199.140 & 68.1 & $1.708 \mathrm{E}-03$ & 22.0 & 25 & & \\
\hline 2 & 12 & -1 & 2 & 13 & 11 & 1 & 2 & 12 & 221199.156 & 68.1 & $1.718 \mathrm{E}-03$ & 23.9 & 27 & & \\
\hline 3 & 12 & 8 & 2 & 12 & 11 & 8 & 2 & 11 & 221209.781 & 423.9 & $9.551 \mathrm{E}-04$ & 12.3 & 25 & $2.72(2.0)$ & \\
\hline 3 & 12 & 8 & 2 & 13 & 11 & 8 & 2 & 12 & 221210.140 & 423.9 & $9.634 \mathrm{E}-04$ & 13.4 & 27 & & \\
\hline 3 & 12 & 8 & 2 & 11 & 11 & 8 & 2 & 10 & 221210.172 & 423.9 & $9.537 \mathrm{E}-04$ & 11.3 & 23 & & \\
\hline 4 & 12 & 5 & 1 & 12 & 11 & 5 & 1 & 11 & 221252.937 & 319.3 & $2.844 \mathrm{E}-03$ & 36.6 & 25 & $5.65(2.27)$ & \\
\hline 4 & 12 & 5 & 1 & 13 & 11 & 5 & 1 & 12 & 221252.937 & 319.3 & $2.863 \mathrm{E}-03$ & 39.8 & 27 & & \\
\hline 4 & 12 & 5 & 1 & 11 & 11 & 5 & 1 & 10 & 221252.937 & 319.3 & $2.846 \mathrm{E}-03$ & 33.7 & 23 & & \\
\hline 5 & 12 & 7 & 2 & 12 & 11 & 7 & 2 & 11 & 221265.547 & 330.5 & $2.269 \mathrm{E}-03$ & 29.2 & 25 & 19.98(2.08) & Blended with $\mathrm{CH}_{3} \mathrm{OCHO}$ \\
\hline 5 & 12 & 7 & 2 & 13 & 11 & 7 & 2 & 12 & 221265.547 & 330.5 & $2.288 \mathrm{E}-03$ & 31.8 & 27 & & \\
\hline 5 & 12 & 7 & 2 & 11 & 11 & 7 & 2 & 10 & 221265.547 & 330.5 & $2.272 \mathrm{E}-03$ & 26.9 & 23 & & \\
\hline 6 & 12 & 4 & 1 & 12 & 11 & 4 & 1 & 11 & 221299.875 & 241.8 & $1.532 \mathrm{E}-03$ & 19.7 & 25 & $4.19(4.0)$ & Blended with $\mathrm{CH}_{3} \mathrm{OCHO}$ \\
\hline 6 & 12 & 4 & 1 & 13 & 11 & 4 & 1 & 12 & 221299.875 & 241.8 & $1.540 \mathrm{E}-03$ & 21.4 & 27 & & \\
\hline 6 & 12 & 4 & 1 & 11 & 11 & 4 & 1 & 10 & 221299.875 & 241.8 & $1.529 \mathrm{E}-03$ & 18.1 & 23 & & \\
\hline 7 & 12 & 6 & 2 & 12 & 11 & 6 & 2 & 11 & 221311.953 & 251.2 & $1.291 \mathrm{E}-03$ & 16.6 & 25 & $3.35(3.0)$ & \\
\hline 7 & 12 & 6 & 2 & 13 & 11 & 6 & 2 & 12 & 221311.953 & 251.2 & $1.303 \mathrm{E}-03$ & 18.1 & 27 & & \\
\hline 7 & 12 & 6 & 2 & 11 & 11 & 6 & 2 & 10 & 221311.953 & 251.2 & $1.293 \mathrm{E}-03$ & 15.3 & 23 & & \\
\hline 8 & 12 & 3 & 1 & 12 & 11 & 3 & 1 & 11 & 221338.218 & 178.5 & $1.618 \mathrm{E}-03$ & 20.8 & 25 & $6.28(5.34)$ & Blended with aGg'-( $\left.\mathrm{CH}_{2} \mathrm{OH}\right)_{2}$ \\
\hline 8 & 12 & 3 & 1 & 11 & 11 & 3 & 1 & 10 & 221338.218 & 178.5 & $1.615 \mathrm{E}-03$ & 19.1 & 23 & & \\
\hline 8 & 12 & 3 & 1 & 13 & 11 & 3 & 1 & 12 & 221338.218 & 178.5 & $1.628 \mathrm{E}-03$ & 22.6 & 27 & & \\
\hline 9 & 12 & 5 & 2 & 12 & 11 & 5 & 2 & 11 & 221350.375 & 186.1 & $1.424 \mathrm{E}-03$ & 18.3 & 25 & $4.52(2.42)$ & \\
\hline 9 & 12 & 5 & 2 & 13 & 11 & 5 & 2 & 12 & 221350.375 & 186.1 & $1.433 \mathrm{E}-03$ & 19.9 & 27 & & \\
\hline 9 & 12 & 5 & 2 & 11 & 11 & 5 & 2 & 10 & 221350.375 & 186.1 & $1.421 \mathrm{E}-03$ & 16.8 & 23 & & \\
\hline 10 & 12 & 2 & 1 & 12 & 11 & 2 & 1 & 11 & 221367.672 & 129.5 & $3.354 \mathrm{E}-03$ & 43.1 & 25 & $9.47(4.30)$ & Blended with $\mathrm{A}^{-13} \mathrm{CH}_{3} \mathrm{OH}$ \\
\hline 10 & 12 & 2 & 1 & 11 & 11 & 2 & 1 & 10 & 221367.672 & 129.5 & $3.349 \mathrm{E}-03$ & 39.6 & 23 & & \\
\hline 10 & 12 & 2 & 1 & 13 & 11 & 2 & 1 & 12 & 221367.672 & 129.5 & $3.379 \mathrm{E}-03$ & 46.9 & 27 & & \\
\hline 11 & 12 & 4 & 2 & 12 & 11 & 4 & 2 & 11 & 221380.734 & 135.3 & $3.066 \mathrm{E}-03$ & 39.4 & 25 & 16.77(1.81) & \\
\hline 11 & 12 & 4 & 2 & 11 & 11 & 4 & 2 & 10 & 221380.734 & 135.3 & $3.062 \mathrm{E}-03$ & 36.2 & 23 & & \\
\hline 11 & 12 & 4 & 2 & 13 & 11 & 4 & 2 & 12 & 221380.734 & 135.3 & $3.084 \mathrm{E}-03$ & 42.8 & 27 & & \\
\hline 12 & 12 & 0 & 2 & 11 & 11 & 0 & 2 & 10 & 221394.156 & 74.3 & $1.726 \mathrm{E}-03$ & 20.4 & 23 & $18.99(1.15)$ & Blended with $\mathrm{CH}_{2} \mathrm{DOH}$ \\
\hline 12 & 12 & 0 & 2 & 12 & 11 & 0 & 2 & 11 & 221394.156 & 74.3 & $1.728 \mathrm{E}-03$ & 22.2 & 25 & & \\
\hline 12 & 12 & 0 & 2 & 13 & 11 & 0 & 2 & 12 & 221394.156 & 74.3 & $1.737 \mathrm{E}-03$ & 24.1 & 27 & & \\
\hline 13 & 12 & 3 & 2 & 12 & 11 & 3 & 2 & 11 & 221403.812 & 98.7 & $1.619 \mathrm{E}-03$ & 20.8 & 25 & $7.49(2.09)$ & Blended \\
\hline 13 & 12 & 3 & 2 & 11 & 11 & 3 & 2 & 10 & 221403.812 & 98.7 & $1.616 \mathrm{E}-03$ & 19.1 & 23 & & \\
\hline 13 & 12 & 3 & 2 & 13 & 11 & 3 & 2 & 12 & 221403.812 & 98.7 & $1.629 \mathrm{E}-03$ & 22.6 & 27 & & \\
\hline 14 & 12 & 2 & 2 & 12 & 11 & 2 & 2 & 11 & 221422.375 & 76.3 & $1.674 \mathrm{E}-03$ & 21.5 & 25 & 15.61(7.19) & Blended with $\mathrm{A}-{ }^{13} \mathrm{CH}_{3} \mathrm{OH}$ \\
\hline 14 & 12 & 2 & 2 & 11 & 11 & 2 & 2 & 10 & 221422.375 & 76.3 & $1.676 \mathrm{E}-03$ & 19.8 & 23 & & \\
\hline 14 & 12 & 2 & 2 & 13 & 11 & 2 & 2 & 12 & 221422.375 & 76.3 & $1.687 \mathrm{E}-03$ & 23.4 & 27 & & \\
\hline 15 & 12 & 1 & 2 & 11 & 11 & -1 & 2 & 10 & 221625.906 & 68.2 & $1.715 \mathrm{E}-03$ & 20.2 & 23 & $9.94(2.67)$ & \\
\hline 15 & 12 & 1 & 2 & 12 & 11 & -1 & 2 & 11 & 221625.906 & 68.2 & $1.718 \mathrm{E}-03$ & 22.0 & 25 & & \\
\hline 15 & 12 & 1 & 2 & 13 & 11 & -1 & 2 & 12 & 221625.906 & 68.2 & $1.728 \mathrm{E}-03$ & 23.9 & 27 & & \\
\hline
\end{tabular}

Notes. Exponential notation: $1.469 \mathrm{E}-04=1.469 \times 10^{-4} \cdot{ }^{(1)}$ Index to enumerate the line features in the spectrum (blended lines share the same index). (2) Quantum numbers of the upper and lower levels of the transitions. (3) Rotational energy of the upper level. ${ }^{(4)}$ Einstein coefficient of spontaneous emission. ${ }^{(5)}$ Line strength defined as $\left|\mu_{i j}\right|^{2}=S_{i j} \mu^{2}$ where $\mu$ is the dipole moment. ${ }^{(6)}$ Degeneracy of the upper level. ${ }^{(7)}$ Velocity integrated intensity. 
Table A.5. $\mathrm{CH}_{3} \mathrm{OCHO}$ line parameters.

\begin{tabular}{|c|c|c|c|c|c|c|c|c|c|c|c|c|c|}
\hline \multirow{2}{*}{$N$} & \multicolumn{6}{|c|}{ Quantum numbers: up-low } & \multirow{3}{*}{$\begin{array}{l}v(\mathrm{MHz}) \\
218297.867\end{array}$} & \multirow{3}{*}{$\begin{array}{l}E_{u}(\mathrm{~K}) \\
99.7\end{array}$} & \multirow{2}{*}{$\begin{array}{l}A_{\mathrm{ul}}\left(\mathrm{s}^{-1}\right) \\
v_{t}=0\end{array}$} & \multirow{3}{*}{$\begin{array}{l}S_{i j} \\
16.4\end{array}$} & \multirow{3}{*}{$\begin{array}{l}g u \\
35\end{array}$} & \multirow[t]{2}{*}{$W\left(\mathrm{~K} \mathrm{~km} \mathrm{~s}^{-1}\right)$} & \\
\hline & & & & & & & & & & & & & \\
\hline 1 & 17 & 3 & 14 & 16 & 3 & 13 & & & $1.506 \mathrm{E}-04$ & & & $10.21(8.88)$ & \\
\hline 2 & 17 & 4 & 13 & 16 & 4 & 12 & 220190.266 & 103.1 & $1.522 \mathrm{E}-04$ & 16.1 & 35 & $18.05(2.43)$ & \\
\hline 3 & 33 & 5 & 28 & 33 & 5 & 29 & 220432.932 & 357.4 & $5.710 \mathrm{E}-06$ & 1.16 & 67 & $9.36(3.90)$ & \\
\hline 4 & 17 & -3 & 14 & 16 & -3 & 13 & 218280.838 & 99.1 & $1.507 \mathrm{E}-04$ & 43.6 & 35 & $9.96(2.90)$ & \\
\hline 5 & 17 & -4 & 13 & 16 & -4 & 12 & 220166.852 & 102.6 & $1.522 \mathrm{E}-04$ & 42.9 & 35 & $13.96(0.37)$ & \\
\hline 6 & 18 & 13 & 6 & 17 & 13 & 5 & 221158.521 & 212.4 & 7.789E-05 & 22.9 & 37 & $10.09(3.66)$ & Blended with $\mathrm{CH}_{2} \mathrm{DOH}$ \\
\hline 7 & 18 & 12 & 7 & 17 & 12 & 6 & 221280.890 & 195.8 & $9.063 \mathrm{E}-05$ & 26.6 & 37 & $18.00(4.90)$ & Blended with ${ }^{13} \mathrm{CH}_{3} \mathrm{OH}$ \\
\hline 8 & 18 & -11 & 7 & 17 & -11 & 6 & 221424.615 & 180.6 & $1.024 \mathrm{E}-04$ & 30.0 & 37 & $16.19(4.35)$ & Blended with $\mathrm{CH}_{3} \mathrm{CN} v_{t}=8$ \\
\hline 9 & 18 & 11 & 8 & 17 & 11 & 7 & 221445.622 & 180.6 & $1.025 \mathrm{E}-04$ & 30.0 & 37 & $7.23(3.90)$ & \\
\hline 10 & 18 & -10 & 8 & 17 & -10 & 7 & 221649.374 & 166.7 & $\begin{array}{l}1.134 \mathrm{E}-04 \\
v_{t}=1\end{array}$ & 33.1 & 37 & $6.51(0.65)$ & \\
\hline 1 & 18 & 4 & 15 & 17 & 4 & 14 & 219704.898 & 299.5 & $1.507 \mathrm{E}-04$ & 45.2 & 37 & $4.69(3.67)$ & \\
\hline 2 & 18 & 9 & 9 & 17 & 9 & 8 & 219764.090 & 342.7 & $1.198 \mathrm{E}-04$ & 35.9 & 37 & $6.16(4.18)$ & \\
\hline 3 & 18 & 10 & 9 & 17 & 10 & 8 & 219822.160 & 355.1 & $1.105 \mathrm{E}-04$ & 33.1 & 37 & $8.99(1.76)$ & \\
\hline 3 & 18 & 10 & 8 & 17 & 10 & 7 & 219822.161 & 355.1 & $1.105 \mathrm{E}-04$ & 33.1 & 37 & & \\
\hline 4 & 18 & 9 & 10 & 17 & 9 & 9 & 220030.289 & 342.4 & $1.201 \mathrm{E}-04$ & 35.9 & 37 & $7.60(7.00)$ & \\
\hline 4 & 18 & 9 & 9 & 17 & 9 & 8 & 220030.339 & 342.4 & $1.201 \mathrm{E}-04$ & 35.9 & 37 & & \\
\hline 5 & 18 & -10 & 9 & 17 & -10 & 8 & 220307.810 & 354.8 & $1.115 \mathrm{E}-04$ & 33.1 & 37 & $3.65(0.60)$ & \\
\hline 6 & 18 & 7 & 12 & 17 & 7 & 11 & 220913.784 & 321.3 & $1.375 \mathrm{E}-04$ & 40.6 & 37 & $8.33(4.37)$ & \\
\hline 7 & 18 & -8 & 11 & 17 & -8 & 10 & 221111.015 & 330.7 & $1.308 \mathrm{E}-04$ & 38.5 & 37 & $3.29(2.88)$ & \\
\hline
\end{tabular}

Notes. Same notation as Table A.3.

Table A.6. $\mathrm{CH}_{2} \mathrm{DOH}$ line parameters.

\begin{tabular}{llllllllllllllll}
\hline \hline$N$ & Quantum numbers: up-low & $v(\mathrm{MHz})$ & $E_{u}(\mathrm{~K})$ & $A_{\mathrm{ul}}\left(\mathrm{s}^{-1}\right)$ & $S_{i j}$ & $g u$ & $W\left(\mathrm{~K} \mathrm{~km} \mathrm{~s}^{-1}\right)$ & \\
\hline 1 & 5 & 2 & 4 & 1 & 5 & 1 & 5 & 1 & 218316.390 & 58.7 & $1.818 \mathrm{E}-05$ & 0.83 & 11 & $7.07(1.27)$ & \\
2 & 20 & 5 & 15 & 1 & 19 & 6 & 14 & 0 & 219206.135 & 557.6 & $2.977 \mathrm{E}-05$ & 4.99 & 41 & $2.05(1.16)$ & \\
3 & 5 & 1 & 5 & 0 & 4 & 1 & 4 & 0 & 220071.805 & 35.8 & $2.614 \mathrm{E}-05$ & 2.89 & 11 & $16.05(0.60)$ & Blended with $\mathrm{CH}_{3} \mathrm{OH}$ \\
4 & 17 & 1 & 16 & 0 & 17 & 0 & 17 & 0 & 220552.586 & 335.9 & $7.545 \mathrm{E}-05$ & 10.6 & 35 & $11.74(5.35)$ & \\
5 & 5 & 1 & 5 & 2 & 4 & 1 & 4 & 2 & 221273.004 & 54.7 & $3.371 \mathrm{E}-05$ & 3.66 & 11 & $8.25(3.23)$ & \\
6 & 10 & 1 & 10 & 0 & 9 & 0 & 9 & 1 & 221391.766 & 120.2 & $5.674 \mathrm{E}-05$ & 4.73 & 21 & $21.17(1.58)$ & Blended with $\mathrm{CH}_{3} \mathrm{CN}_{8}=1$ \\
7 & 20 & 2 & 18 & 2 & 20 & 1 & 19 & 2 & 221154.575 & 486.0 & $7.322 \mathrm{E}-05$ & 12.0 & 41 & $13.20(2.10)$ & Blended with methyl formate \\
8 & 6 & 2 & 5 & 1 & 6 & 1 & 6 & 1 & 221178.153 & 71.6 & $2.067 \mathrm{E}-05$ & 1.07 & 13 & $12.48(5.20)$ & Blended with HDCS \\
\hline
\end{tabular}

Notes. Same notation as Table A.3.

Table A.7. HNCO line parameters.

\begin{tabular}{lllllllllllll}
\hline \hline$N$ & \multicolumn{6}{c}{ Quantum numbers: up-low } & $v(\mathrm{MHz})$ & $E_{u}(\mathrm{~K})$ & $A_{\mathrm{ul}}\left(\mathrm{s}^{-1}\right)$ & $S_{i i}$ & $g u$ & $W\left(\mathrm{~K} \mathrm{~km} \mathrm{~s}^{-1}\right)$ \\
\hline 1 & 10 & 1 & 10 & 9 & 1 & 9 & 218981.009 & 101.1 & $1.462 \mathrm{E}-04$ & 9.79 & 21 & $22.71(1.42)$ \\
2 & 10 & 3 & 8 & 9 & 3 & 7 & 219656.769 & 433.0 & $1.235 \mathrm{E}-04$ & 8.19 & 21 & $10.68(1.48)$ \\
2 & 10 & 3 & 7 & 9 & 3 & 6 & 219656.771 & 433.0 & $1.235 \mathrm{E}-04$ & 8.19 & 21 & \\
3 & 10 & 2 & 9 & 9 & 2 & 8 & 219733.850 & 228.3 & $1.383 \mathrm{E}-04$ & 9.17 & 21 & $27.10(1.89)$ \\
3 & 10 & 2 & 8 & 9 & 2 & 7 & 219737.193 & 228.3 & $1.383 \mathrm{E}-04$ & 9.17 & 21 & \\
4 & 10 & 0 & 10 & 9 & 0 & 9 & 219798.274 & 58.0 & $1.510 \mathrm{E}-04$ & $1.00 \mathrm{E}+01 \mathrm{a}$ & 21 & $27.85(4.74)$ \\
5 & 10 & 1 & 9 & 9 & 1 & 8 & 220584.751 & 101.5 & $1.494 \mathrm{E}-04$ & $9.79 \mathrm{E}+00 \mathrm{a}$ & 21 & $22.70(4.17)$ \\
\hline
\end{tabular}

Notes. Same notation as Table A.3. 
Table A.8. aGg' $-\left(\mathrm{CH}_{2} \mathrm{OH}\right)_{2}$ line parameters.

\begin{tabular}{|c|c|c|c|c|c|c|c|c|c|c|c|c|c|c|c|}
\hline \multicolumn{2}{|l|}{$N$} & \multicolumn{7}{|c|}{ Quantum numbers: up-low } & \multirow{2}{*}{$\begin{array}{l}v(\mathrm{MHz}) \\
218371.495\end{array}$} & \multirow{2}{*}{$\begin{array}{l}E_{u}(\mathrm{~K}) \\
132.7\end{array}$} & \multirow{2}{*}{$\frac{A_{\mathrm{ul}}\left(\mathrm{s}^{-1}\right)}{1.881 \mathrm{E}-04}$} & \multirow{2}{*}{$\frac{S_{i j}}{145}$} & \multirow{2}{*}{$\frac{g u}{405}$} & \multicolumn{2}{|l|}{$W\left(\mathrm{~K} \mathrm{~km} \mathrm{~s}^{-1}\right)$} \\
\hline 1 & 22 & 4 & 19 & 0 & 21 & 4 & 18 & 1 & & & & & & $3.07(3.00)$ & \\
\hline 2 & 22 & 15 & 7 & 0 & 21 & 15 & 6 & 1 & 218379.983 & 234.8 & $1.355 \mathrm{E}-04$ & 81.4 & 315 & $2.21(0.17)$ & \\
\hline 2 & 22 & 15 & 8 & 0 & 21 & 15 & 7 & 1 & 218379.983 & 234.8 & $1.355 \mathrm{E}-04$ & 105 & 405 & & \\
\hline 3 & 22 & 13 & 9 & 0 & 21 & 13 & 8 & 1 & 218574.680 & 207.3 & $1.652 \mathrm{E}-04$ & 98.9 & 315 & $8.35(2.65)$ & ne? \\
\hline 3 & 22 & 13 & 10 & 0 & 21 & 13 & 9 & 1 & 218574.680 & 207.3 & $1.651 \mathrm{E}-04$ & 127 & 405 & & \\
\hline 4 & 22 & 11 & 12 & 0 & 21 & 11 & 11 & 1 & 112 & 183.8 & $1.911 \mathrm{E}-04$ & 147 & 405 & $3.90(0.15)$ & \\
\hline 4 & 22 & 11 & 11 & 0 & 21 & 11 & 10 & 1 & .112 & 183.8 & $1.911 \mathrm{E}-04$ & 114 & 315 & & \\
\hline 5 & 22 & 10 & 13 & 0 & 21 & 10 & 12 & 1 & 219089.720 & 173.5 & $2.027 \mathrm{E}-04$ & 155 & 405 & $5.06(0.49)$ & Blended with $\mathrm{CH}_{3} \mathrm{OCHO}$ \\
\hline 5 & 22 & 10 & 12 & 0 & 21 & 10 & 11 & 1 & 219089.727 & 173.5 & $2.027 \mathrm{E}-04$ & 121 & 315 & & \\
\hline 6 & 26 & 11 & 16 & 1 & 26 & 10 & 16 & 0 & 219384.910 & 232.5 & $5.221 \mathrm{E}-06$ & 71.4 & 371 & $4.03(0.05)$ & Well below the fit! \\
\hline 6 & 22 & 9 & 14 & 0 & 21 & 9 & 13 & 1 & 219385.177 & 164.3 & $2.136 \mathrm{E}-04$ & 163 & 405 & & \\
\hline 6 & 26 & 11 & 15 & 1 & 26 & 10 & 17 & 0 & 219385.324 & 232.5 & $5.222 \mathrm{E}-06$ & 91.7 & 477 & & \\
\hline 6 & 22 & 9 & 13 & 0 & 21 & 9 & 12 & 1 & 219385.425 & 164.3 & $2.136 \mathrm{E}-04$ & 127 & 315 & & \\
\hline 7 & 22 & 1 & 21 & 1 & 21 & 1 & 20 & 0 & 219580.671 & 122.2 & $2.568 \mathrm{E}-04$ & 195 & 405 & $12.63(0.56)$ & Ble \\
\hline 9 & 20 & 4 & 16 & 1 & 19 & 4 & 15 & 0 & 219764.925 & 114.4 & $2.453 \mathrm{E}-04$ & 169 & 369 & $9.50(0.60)$ & Blended with $\mathrm{CH}_{3} \mathrm{OCHO}$ \\
\hline 9 & 21 & 4 & 18 & 1 & 20 & 4 & 17 & 0 & 221007.823 & 122.6 & $2.657 \mathrm{E}-04$ & 189 & 387 & $5.67(0.28)$ & \\
\hline 10 & 22 & 6 & 17 & 0 & 21 & 6 & 16 & 1 & 221038.799 & 142.6 & 2.395E-04 & 178 & 405 & $3.77(0.42)$ & \\
\hline 11 & 22 & 5 & 18 & 0 & 21 & 5 & 17 & 1 & 221100.315 & 137.4 & $2.368 \mathrm{E}-04$ & 176 & 405 & $3.39(0.26)$ & \\
\hline 12 & 23 & 3 & 21 & 0 & 22 & 3 & 20 & 1 & 221338.974 & 138.4 & $2.717 \mathrm{E}-04$ & 164 & 329 & $7.19(0.075)^{*}$ & Blended with $\mathrm{CH}_{3} \mathrm{CN} v_{8}=1$ \\
\hline
\end{tabular}

Notes. Same notation as Table A.3. ${ }^{(*)}$ In Fig. 2, we have subtracted the $\mathrm{CH}_{3} \mathrm{CN} v_{8}=1$ contribution that accounts for $\sim 50 \%$ of the flux. 